\title{
ORIGEN Y SUSTENTO DEL AGOTAMIENTO DE LOS DERECHOS DE PROPIEDAD INTELECTUAL*
}

\section{ORIGIN AND BASIS OF THE INTELLECTUAL PROPERTY RIGHTS EXHAUSTION}

\author{
Juan Camilo Contreras-Jaramillo** \\ Fecha de recepción: 23 de julio de 2015 \\ Fecha de aceptación: 20 de septiembre de 2015 \\ Disponible en linea: 30 de noviembre de 2015
}

\section{Para citar este artículo/To cite this article}

\author{
Contreras-Jaramillo, Juan Camilo, Origen y sustento del agotamiento \\ de los derechos de propiedad intelectual, 131 Vniversitas, 277-322 (2015). \\ http://dx.doi.org/10.11144/Javeriana.vj131.osad \\ doi:10.11144/Javeriana.vj131.osad
}

* Artículo de revisión elaborado en el marco del Doctorado en Ciencias Jurídicas de la Pontificia Universidad Javeriana y el proyecto de investigación Limitaciones y restricciones a la propiedad, del Departamento de Derecho Privado de la Facultad de Ciencias Jurídicas de la Pontificia Universidad Javeriana.

** Profesor del Departamento de Derecho Privado, Facultad de Ciencias Jurídicas, Pontificia Universidad Javeriana. Estudiante del doctorado en ciencias jurídicas, Pontificia Universidad Javeriana. Contacto: juancontreras@javeriana.edu.co 


\section{RESUMEN}

Las tensiones que actualmente sufre la figura del agotamiento de los derechos de propiedad intelectual se han caracterizado como una pugna entre viejas y nuevas concepciones del sistema de regulación de la propiedad intelectual; sin embargo, estas no son nada distinto a las expresiones actuales de la antigua convivencia entre las explicaciones de propiedad y de mercado. Este artículo de revisión busca identificar el origen de las teorías que explican la figura del agotamiento en Estados Unidos, la Unión Europea y Colombia, con el objetivo de analizar su incidencia en las actuales tensiones de este concepto.

\section{Palabras clave}

agotamiento; primera venta; derechos de propiedad intelectual; competencia; mercados; propiedad 


\section{ABSTRACT}

Today's debates around the exhaustion doctrine it's characterized by the confrontation between old and new conceptions of the intellectual property system. Notwithstanding, such tensions do not differ from the longstanding coexistence between the property and market rules. This review article aims to identify the origin of the exhaustion's explanatory theories in the United States, the European Union and Colombia targeting the current tensions of this figure.

\section{Keywords}

exhaustion doctrine; first sale doctrine; intellectual property; competition law; market; property

\section{SUMARIO}

Introducción.- I. Primera parte.- A. Origen de la figura del agotamiento de los derechos de propiedad intelectual: el origen jurisprudencial del agotamiento del derecho.- 1. El agotamiento y la sucesión entre propiedad y competencia en Estados Unidos.- 2. El caso de los procesos de integración en la Unión Europea.- 3. La teoría inconducente del caso colombiano.- 4. ¿El agotamiento del derecho: una figura de origen local o internacional?- II. SEgUNDA PARTE.- A . Las teorías detrás del agotamiento.- 1. El juego de las justificaciones.- 2. Las teorías frente a las tensiones del agotamiento.- 2.1. El objeto del agotamiento.- 2.2. Derechos que se agotan.- ConClusiones.- BibliogRAfía. 


\section{INTRODUCCIÓN}

El agotamiento de los derechos de propiedad intelectual (DPI $)^{1}$ ha sido definido, por ejemplo, como un "principio según el cual, una vez que un producto ha sido vendido en un mercado, el titular de la propiedad intelectual ya no tiene derechos sobre él (...)"2. Sin embargo, la simpleza de la definición oculta el entramado de teorías, intervenciones judiciales y regulaciones especiales que conforman la figura del agotamiento de los DPI, pues se enfrentan e interrelacionan los diferentes derechos de propiedad (los límites y excepciones a tales derechos), las relaciones contractuales (los comerciantes y consumidores involucrados en ellas) y la protección del mercado (los eventuales actos de competencia desleal, la promoción de la libre competencia, la eficiencia y la integración de los mercados).

Este estudio pretende identificar las distintas explicaciones y teorías que soportan esta figura, conocida como agotamiento del derecho, doctrina del consentimiento o doctrina de la primera venta ${ }^{3}$. Revisaremos sus orígenes, su desarrollo y los efectos derivados de su aplicación desde la perspectiva que brindan los marcos teóricos que han servido como sustento de la regulación del agotamiento de los derechos de propiedad intelectual.

Para esta tarea, se han escogido tres referentes geográficos sobre los que se realizará el estudio planteado: la Unión Europea (UE), Estados Unidos de América (EEUU) y Colombia.

Concretamente, en este trabajo de revisión, se toma uno de los muchos elementos que integran el gran sistema de protección de la propiedad intelectual con el objetivo de analizarlo, identificar sus justificaciones, características y retos a futuro.

1 Algunas veces en el texto se utilizará la expresión completa de Derechos de Propiedad Intelectual y en otras oportunidades su abreviatura DPI, para facilitar la lectura y evitar cacofonías y repeticiones disonantes en el escrito.

2 Organización Mundial de Propiedad Intelectual, OMPI, Glosario de términos. Disponible en: http://www.wipo.int/wipolex/en/glossary/\#O. Organización Mundial del Comercio, OMC, Glosario de términos. Disponible en: http://www.wto.org/spanish/thewto_s/glossary_s/ glossary_s.htm, https://www.wto.org/english/thewto_e/glossary_e/glossary_e.htm. Estados Unidos, Copyright Act, 17 USCS Sects. 101-810, 1976, art. 109. Disponible en: http://www. copyright.gov/title17/, http://copyright.gov/title17/92appa.pdf, https://www.law.cornell.edu/ copyright/copyright.table.html

3 En el presente trabajo se utilizará la palabra agotamiento para referirse al fenómeno tanto en materia de propiedad industrial, como en derecho de autor. En su desarrollo se puntualizarán los diferentes efectos que estas denominaciones pueden conllevar. 


\section{PRIMERA PARTE}

Aunque el lector podrá identificar en la mayoría de los casos diferentes teorías de la propiedad (incluso se harán algunas referencias directas en el texto a ellas), este artículo no pretende asumir las discusiones sobre posiciones filosóficas, políticas o económicas ${ }^{4}$ en torno a esta o a la propiedad intelectual en particular ${ }^{5}$. La primera parte de este trabajo está dirigida a una finalidad mucho más modesta: analizar la creación y desarrollo de la figura del agotamiento de los DPI y las teorías que han explicado su surgimiento, consolidación y avances; esto, con el fin de concretar un vocabulario común que permita adelantar un conjunto de reflexiones sobre la figura del agotamiento de los DPI, sin trasladarse a los enfrentamientos de posiciones inconmensurables sobre el deber ser de la propiedad intelectual $^{6}$.

\section{A. Origen de la figura del agotamiento de los derechos de propiedad intelectual: el origen jurisprudencial del agotamiento del derecho}

En Estados Unidos ${ }^{7}$ y la Unión Europea ${ }^{8}$ se ha reconocido invariablemente el origen jurisprudencial del agotamiento de los DPI; bien como un límite o excepción propia del derecho de propiedad intelectual, o bien como un instrumento regulador del mercado. El carácter netamente jurisprudencial de la regla de la "primera venta" o teoría del agotamiento del derecho se puede identificar claramente desde finales del siglo XIX, iniciando en Estados Unidos (al igual que en Alemania, como se señalará más adelante) con las

4 Sobre las justificaciones económicas, Ronald A. Cass \& Keith N. Hylton, The Laws of Creation. Property Rights in the World of Ideas (Harvard University Press, Boston, 2013). O su contrapunto, William Patry, How to Fix Copyright (Oxford University Press, Oxford, 2011).

5 William Fisher destaca los "factores culturales e ideológicos" que dieron pie al desarrollo de la propiedad intelectual. WiLliam W. Fisher, III, The Growth of Intellectual Property: A History of the Ownership of Ideas in the United States/Geistiges Eigentum - ein ausufernder Rechtsbereich: Die Geschichte des Ideenschutzes in den Vereinigten Staaten, Eigentum im Internationalen Vergleich, 265-291 (Vandenhoeck \& Ruprecht, 1999). Disponible en: http:// cyber.law.harvard.edu/people/tfisher/iphistory.pdf

6 En este sentido, ver el propósito de los Midlevel Principles que plantea Robert P. Merges, Justifying Intellectual Property (Harvard University Press, Cambridge, London, 2011).

7 Christina Bohannan \& Herbert HovenKamp, Creation without Restraint. Promoting Liberty and Rivalry in Innovation, 368 (Oxford University Press, Oxford, 2012).

8 Guy Tritton, Intellectual Property in Europe, 648 ( ${ }^{\text {rd }}$ ed., Sweet \& Maxwell, London, 2008). 
primeras sentencias que reconocieron esta figura ${ }^{9}$, pasando luego por las decisiones del Tribunal de Justicia de la Unión Europea (llamado Tribunal de Justicia de las Comunidades Europeas hasta 2009) en 1971 sobre los procesos de integración económica que se estaban consolidando en el viejo continente ${ }^{10}$, para terminar con los más recientes pronunciamientos sobre la aplicación de la figura a soportes digitales ${ }^{11}$ y contratos de licencia.

\section{El agotamiento y la sucesión entre propiedad y com- petencia en Estados Unidos}

El agotamiento de los DPI en Estados Unidos surge del análisis jurisprudencial que hace la Corte Suprema de la relación existente entre el monopolio legal otorgado al titular de una patente y el derecho real de dominio de quien adquiere un aparato contentivo de esa misma patente. Así, en el caso Adams v. Burke, de $1873^{12}$, la Corte Suprema de Estados Unidos estudió la demanda instaurada por un cesionario de una patente sobre tapas de ataúdes contra el dueño de una funeraria que utilizaba la patente dentro del territorio asignado de forma exclusiva por el titular de la patente al demandante; sin embargo, el análisis de la Corte Suprema se concentró en determinar si este uso por parte de la funeraria constituía una infracción al derecho del cesionario teniendo en cuenta que el propietario de la funeraria había comprado legítimamente de otro cesionario las tapas protegidas por el título de patente, fuera del territorio asignado al demandante.

En este caso, la Corte Suprema reconoció que, aunque el Patent Act de $1836^{13}$, vigente para ese momento, otorgaba al titular del

9 Antoni Rubi-Puig, Copyright Exhaustion Rationales and Used Software: A Law and Economics Approach to Oracle v. UsedSoft, 4 Journal of Intellectual Property, Information Technology and e-Commerce Law, JIPITEC, 3, 159-178 (2013). Disponible en: http://papers.ssrn.com/sol3/ papers.cfm?abstract_id=2408659. Yonatan Even, Appropriability, First Sale \& Exhaustion, 1-77, 5-10 (September 28, 2008). Disponible en: http://ssrn.com/abstract $=1274822$

10 Guy Tritton, Intellectual Property in Europe, 648 ( $3^{\text {rd }}$ ed., Sweet \& Maxwell, London, 2008).

11 Antoni Rubí-Puig, Copyright Exhaustion Rationales and Used Software: A Law and Economics Approach to Oracle v. UsedSoft, 4 Journal of Intellectual Property, Information Technology and e-Commerce Law, JIPITEC, 3, 159-178, 170 (2013). Disponible en: http://papers.ssrn.com/sol3/ papers.cfm?abstract_id $=2408659$

12 Estados Unidos, Corte Suprema, Adams v. Burke, 84 U.S. 453 (1873). Disponible en: https:// supreme.justia.com/cases/federal/us/84/453/case.html

13 Estados Unidos, Patent Act of 1836, Ch. 357, 5 Stat. 117 (July 4, 1836). Disponible en: http:// www.ipmall.info/hosted_resources/lipa/patents/patent_act_of_1836.pdf 
derecho de patente (vía su cesionario en este caso) un "monopolio" sobre el uso, la fabricación y cualquier autorización a terceros sobre la patente o parte de ella, y que incluso permitía otorgar autorizaciones para la explotación y uso de la patente en todo el territorio nacional o en parte de él; tal disposición, en el caso de la subdivisión de territorios con fines comerciales por parte del titular de la patente, debe ser interpretada bajo la regla de que "una vez fabricados y vendidos legítimamente, los objetos protegidos por una patente no son sujetos de restricción alguna respecto del uso que de ellos se haga"14. Para llegar por primera vez a la regla que acepta el agotamiento de un derecho de propiedad intelectual, la Corte Suprema de Estados Unidos parte del reconocimiento inicial de la teoría de la propiedad absoluta ${ }^{15}$, mediante la cual la jurisprudencia hasta ese entonces sostuvo que por medio de la venta de una "máquina" patentada, el comprador adquiría el derecho de uso sobre esa máquina; sin embargo, inmediatamente fue expuesta esta línea jurisprudencial, la Corte asumió como justificación de la regla del agotamiento del derecho la "teoría de la recompensa"16. Así, la Corte afirmó que con la primera venta de una "máquina o instrumento" patentado, el titular de los derechos recibe toda la "consideración" económica que la ley puede otorgar ${ }^{17}$; es decir, se estableció jurisprudencialmente hasta dónde llega el derecho de propiedad del titular de la patente.

La teoría de la recompensa está fundada en la más general labor-desert theory sobre la propiedad en general ${ }^{18}$, mediante la cual se defiende el merecimiento de una persona a poseer algo que

14 Estados Unidos, Corte Suprema, Adams v. Burke, 84 U.S. 453 (1873). Disponible en: https:// supreme.justia.com/cases/federal/us/84/453/case.html. Traducción libre del autor.

15 Antoni Rubí-Puig, Copyright Exhaustion Rationales and Used Software: A Law and Economics Approach to Oracle v. UsedSoft, 4 Journal of Intellectual Property, Information Technology and e-Commerce Law, JIPITEC, 3, 159-178, 162 (2013). Disponible en: http://papers.ssrn.com/sol3/ papers.cfm?abstract_id=2408659

16 Antoni Rubí-Puig identifica esta teoría con el reconocimiento de ganancias a favor del titular de DPI, mediante el ejercicio de los actos legalmente reconocidos. ANTONI Rubí-Puig, Copyright Exhaustion Rationales and Used Software: A Law and Economics Approach to Oracle v. UsedSoft, 4 Journal of Intellectual Property, Information Technology and e-Commerce Law, JIPITEC, 3, 159-178 (2013). Disponible en: http://papers.ssrn.com/sol3/papers.cfm?abstract_id=2408659

17 "The patentee or his assignee having in the act of sale received all the royalty or consideration which he claims for the use of his invention in that particular machine or instrument".

18 Según Peter Jaszi, esta teoría ayudó a forjar las ideas fundantes de la propiedad intelectual que se mantienen intactas hoy. PETER JASZI, Toward a Theory of Copyright: The Metamorphoses of 'Authorship', 1991 Duke Law Journal, 455-502, 478 (1991). Disponible en: http://scholarship. law.duke.edu/cgi/viewcontent.cgi?article $=3150 \&$ context $=\mathrm{dlj}$ 
el titular de la patente ha creado mediante su labor productiva ${ }^{19}$. Así el agotamiento se identifica como un límite intrínseco a la propiedad intelectual, mediante el cual se logra evitar que el titular del derecho ${ }^{20}$ obtenga más provecho del que debería recibir por su esfuerzo creador.

Esta doctrina utilitarista que fue ratificada en el caso Keeler $v$. Standard Folding Bed Co., de $1895^{21}$, terminó de consolidarse como precedente en Estados Unidos, para derecho de autor en $1908^{22}$ y en 1913 para asuntos relacionados con patentes ${ }^{23}$.

Justo cuando el agotamiento de los DPI comenzaba a consolidarse en la jurisprudencia de la Corte Suprema de Estados Unidos, la sentencia de la Corte de Apelaciones del 6 Circuito, en el caso Heaton-Peninsular Button-Fastener Co. v. Eureka Specialty Co. $(1896)^{24}$, sugiere por primera vez la relación entre la extensión del derecho de propiedad intelectual, los contratos comerciales y el mercado. En esa sentencia, tal vez bajo la influencia de la norma

19 William W. Fisher, III, The Growth of Intellectual Property: A History of the Ownership of Ideas in the United States/Geistiges Eigentum - ein ausufernder Rechtsbereich: Die Geschichte des Ideenschutzes in den Vereinigten Staaten, Eigentum im Internationalen Vergleich, 265-291 (Vandenhoeck \& Ruprecht, 1999). Disponible en: http://cyber.law.harvard.edu/people/tfisher/ iphistory.pdf

20 Se entiende como aquella limitación que tiene su fundamento en la misma finalidad del derecho; en este caso, derecho de propiedad intelectual. Esta se contrapone a una limitación extrínseca. ERnesto Peña-Quiñonez, El derecho de bienes (1 $1^{\text {a }}$ ed., Librería Jurídicas Wilches, Bogotá, 1995). Disponible en: http://www.dasumo.com/libros/el-derecho-de-bienes- $\%$ E2\%80\%93ernesto-pe $\% \mathrm{C} 3 \% \mathrm{~B} 1 \mathrm{a}-$ qui $\% \mathrm{C} 3 \% \mathrm{~B} 1$ onez-pdf.html. Sobre el agotamiento entendido como un límite intrínseco de los DPI, FERNANDO CARBAJO-CASCÓN, La distribución selectiva y el comercio paralelo de productos de lujo (1ª ed., Grupo Editorial Ibáñez, Bogotá, 2010).

21 Estados Unidos, Corte Suprema, Keeler v. Standard Folding Bed Co., 157 U.S. 659 (1895). Disponible en: https://supreme.justia.com/cases/federal/us/157/659/case.html

22 Yonatan Even, Appropriability, First Sale \& Exhaustion, 1-77 (September 28, 2008). Disponible en: http://ssrn.com/abstract=1274822. Casos como Estados Unidos, Corte de Apelaciones del 8 Circuito, National Phonograph Co. v. Schlegel, 128 F. 733 (US Court of Appeals for the Eighth Circuit, $8^{\text {th }}$ Cir. 1904) y Estados Unidos, Corte Suprema, Bobbs-Merrill Co. v. Straus, 201 U.S. 339 (1908). Disponible en: http://caselaw.findlaw.com/us-supreme-court/210/339.html

23 Yonatan Even, Appropriability, First Sale \& Exhaustion, 1-77 (September 28, 2008). Disponible en: http://ssrn.com/abstract=1274822. Casos como Estados Unidos, Corte Suprema, E. Bement \& Sons v. National Harrow Co., 186 U.S. 70 (1902). Disponible en: https://www.law.cornell. edu/supremecourt/text/186/70. Estados Unidos, Corte de Apelaciones del 6 Circuito, John D. Park \& Sons Co. v. Hartman, 153 F. 24, 27 (US Court of Appeals for the Sixth Circuit, $6^{\text {th }}$ Cir. 1907). Estados Unidos, Corte de Apelaciones del 7 Circuito, Victor Talking Machine Co. v. The Fair, 123 F. 428 (US Court of Appeals for the Seventh Circuit, $7^{\text {th }}$ Cir. 1903). Estados Unidos, Corte de Apelaciones del 8 Circuito, National Phonograph Co. v. Schlegel, 128 F. 733 (US Court of Appeals for the Eighth Circuit, $8^{\text {th }}$ Cir. 1904).

24 Estados Unidos, Corte de Apelaciones del 6 Circuito, Heaton-Peninsular Button-Fastener Co. v. Eureka Specialty Co., 77 F. 288 (US Court of Appeals for the Sixth Circuit, $6^{\text {th }}$ Cir. 1896). Disponible en: http://openjurist.org/77/f1d/288 
federal fundante de la libre competencia en Estados Unidos, la Sherman Act, de $1890^{25}$ (conocida como la legislación antitrust), se buscaba responder a la necesidad de crear un límite mediante el agotamiento a los efectos restrictivos de la libre competencia que los acuerdos posventa ${ }^{26}$ de productos podían tener en el mercado. A pesar de la introducción de una explicación de mercado, cercana a la protección de la competencia, durante casi un siglo la teoría de la recompensa, vinculada directamente con el derecho de propiedad, se mantuvo predominante en la jurisprudencia de la Corte Suprema de Estados Unidos ${ }^{27}$; solo hasta $1990^{28}$ se puede evidenciar la irrupción completa de la doctrina de la protección de la competencia en la regla jurisprudencial ${ }^{29}$.

25 Yonatan Even, Appropriability, First Sale \& Exhaustion, 1-77, 23 (September 28, 2008). Disponible en: http://ssrn.com/abstract=1274822. Estados Unidos, Sherman Act, 15 USC, Sects. 1-7, 1890. Disponible en: http://www.ourdocuments.gov/doc.php?doc=51\&page=transcript

26 Para ampliar sobre la relación del derecho de competencia en la jurisprudencia de Estados Unidos en materia de agotamiento del derecho, Her Bert J. HovenKAMP, Innovation and Competition Policy, Ch. 10 (2 ${ }^{\text {nd }}$ ed.): Post-Sale and Related Distribution Restraints Involving IP Rights, Cases and Materials on Innovation and Competition Policy, Chapter 10 (2013). Disponible en: http://papers.ssrn.com/sol3/papers.cfm?abstract_id=1949392. Sobre los acuerdos posventa y los sistemas de distribución comercial en general, Osvaldo J. Marzorati, Sistemas de distribución comercial. Contratos de agencia, distribución, concesión y franchising. Modalidades de instrumentación. Normativa aplicable. Derechos y obligaciones de las partes. Renuncia. Rescisión. Solidaridad laboral. Defensa de la competencia. Internet. Propiedad industrial. Criterios jurisprudenciales. Tendencias en el derecho extranjero ( $3^{\mathrm{a}} \mathrm{ed}$. , Editorial Astrea, Buenos Aires, 2011). Fernando CARBAJo-CASCón, La distribución selectiva y el comercio paralelo de productos de lujo (1 $1^{\mathrm{a}}$ ed., Grupo Editorial Ibáñez, Bogotá, 2010).

27 Esto se evidencia en decisiones como Estados Unidos, Corte Suprema, U.S. v. Univis Lens Co., 316 U.S. 241 (1942). Disponible en: https://supreme.justia.com/cases/federal/us/316/241/case. html. Estados Unidos, Corte Suprema, U.S. v. Masonite Corporation, 316 U.S. 265, 277-278 (1942). Disponible en: https://supreme.justia.com/cases/federal/us/316/265/case.html. Estados Unidos, Corte Suprema, U.S. v. Line Material Co., 333 U.S. 287, 300 (1948). Disponible en: https://supreme.justia.com/cases/federal/us/333/287/. Estados Unidos, Corte Suprema, Aro Manufacturing Co. v. Convertible Top Replacement Co., 365 U.S. 336, 259 (1961). Disponible en: https://supreme.justia.com/cases/federal/us/365/336/case.html

28 El caso Mallinckrodt, Inc. v. Medipart, Inc. involucraba la venta de nebulizadores patentados a hospitales. Sobre tales nebulizadores el fabricante había dejado la inscripción "para un único uso", pero el demandado Medipart reacondicionaba los nebulizadores usados. Finalmente, en este caso, la Corte de Apelaciones del Circuito decidió que no se constituyó una infracción de la patente, pues dentro de su análisis revisó que la restricción posventa conllevaba efectos anticompetitivos que exceden el derecho de patente otorgado. Estados Unidos, Corte Federal del Distrito Norte de Illinois, Mallinckrodt, Inc. v. Medipart, Inc., No. 89-C-4524 (1992 - WL 19535 (Northern District of Illinois Judges, Feb. 16, 1990).

29 No obstante, mucho antes, en el caso Bobbs-Merrill v. Straus (1908), algunos identifican el germen del vínculo entre el agotamiento y la protección a la competencia. ArIEL KATZ, What Antitrust Law Can (and Cannot) Teach About the First Sale Doctrine (January 23, 2012). Disponible en: http://papers.ssrn.com/sol3/papers.cfm?abstract_id=1845842. Estados Unidos, Corte Suprema, Bobbs-Merrill Co. v. Straus, 201 U.S. 339 (1908). Disponible en: http://caselaw. findlaw.com/us-supreme-court/210/339.html 
Esta última explicación del agotamiento de los DPI ${ }^{30}$ trajo consigo la yuxtaposición de tres aspectos que no eran objeto de un análisis conjunto bajo las explicaciones de la propiedad absoluta o de la teoría de la recompensa: (i) los derechos de propiedad intelectual; (ii) las relaciones contractuales entre las partes; y (iii) los efectos de los dos anteriores en el mercado ${ }^{31}$.

Incluso quienes critican la intervención del derecho de la competencia en la figura del agotamiento, han reconocido que tras la figura del agotamiento del derecho de los DPI juegan los contratos y el mercado como factores relevantes ${ }^{32}$. A pesar de manifestar tal reconocimiento, quienes critican tal relación extrañan aquel período en que la jurisprudencia distinguía las diferentes categorías legales, separando los efectos de la aplicación del derecho de propiedad, por un lado; y por el otro, el derecho de contratos y la protección a la competencia ${ }^{33}$.

Tal interrelación normalmente se explica desde las restricciones posventa de mercancías, entendidas como cualquier condicionamiento impuesto por el vendedor respecto del uso o reventa del bien vendido originalmente ${ }^{34}$. Sin embargo, el efecto del nuevo entendimiento sobre las restricciones posventa, es decir, el acercamiento de las tres categorías jurídicas (propiedad intelectual, relaciones

30 Yonatan Even, Appropriability, First Sale \& Exhaustion, 1-77, 21 (September 28, 2008). Disponible en: http://ssrn.com/abstract $=1274822$

31 Sobre la integración de los tres elementos, Herbert J. Hovenkamp, Post-Sale Restraints and Competitive Harm: The First Sale Doctrine in Perspective, NYU Annual Survey of American Law, 101-158 (2011). Disponible en: http://papers.ssrn.com/sol3/papers.cfm?abstract_id=1540527\#\#. William W. Fisher, III, The Growth of Intellectual Property: A History of the Ownership of Ideas in the United States/Geistiges Eigentum - ein ausufernder Rechtsbereich: Die Geschichte des Ideenschutzes in den Vereinigten Staaten, Eigentum im Internationalen Vergleich, 265-291 (Vandenhoeck \& Ruprecht, 1999). Disponible en: http://cyber.law.harvard.edu/people/tfisher/ iphistory.pdf

32 Melissa Goldberg, A Textbook Dilemma: Should the First Sale Doctrine Provide a Valid Defense for Foreign-Made Goods?, 81 Fordham Law Review, 101-136 (2012). Disponible en: http:// ssrn.com/abstract $=2042811$

33 Ariel Katz, What Antitrust Law Can (and Cannot) Teach About the First Sale Doctrine, 5 (January 23, 2012). Disponible en: http://papers.ssrn.com/sol3/papers.cfm?abstract_id=1845842. Yonatan Even, Appropriability, First Sale \& Exhaustion, 1-77, 7 (September 28, 2008). Disponible en: http://ssrn.com/abstract=1274822. Yonatan Even afirma: Al agregarse el razonamiento de la competencia "se está eliminando de la discusión la doctrina misma del agotamiento (...) y se les otorga todo el peso argumentativo a los efectos anticompetitivos". Herbert J. Hovenkamp, Resale Price Maintenance: Consignment Agreements, Copyrighted or Patented Products and the First Sale Doctrine (University of Iowa Legal Studies Research Paper, 10-42, 2010). Disponible en: http://papers.ssrn.com/sol3/papers.cfm?abstract_id=1671943

34 Christina Bohannan \& Herbert Hovenkamp, Creation without Restraint. Promoting Liberty and Rivalry in Innovation, 365 (Oxford University Press, Oxford, 2012). 
contractuales y libre mercado) es utilizado por la doctrina para explicar la correlación inherente entre estas tres categorías ${ }^{35} \mathrm{o}$, por el contrario, para evidenciar el decaimiento de la teoría del agotamiento del derecho ${ }^{36}$.

Estas dos visiones respecto de las restricciones posventa (o restricciones verticales, bien sean intermarcas o intramarcas ${ }^{37}$ ) surgen desde un punto en común, ambas reconocen que el derecho de la competencia moderno sugiere que tales restricciones no generan problemas de competencia relevantes en la generalidad de los casos, es decir, que este ordenamiento entiende las restricciones verticales como benignas en la mayoría de los $\operatorname{casos}^{38}$.

Los críticos de la inter relación entre las tres categorías jurídicas (propiedad intelectual, relaciones contractuales y libre mercado) antes mencionadas aseguran que ante la mirada complaciente que el derecho de la competencia les da a las restricciones posventa, la teoría del agotamiento del derecho hoy es sostenida por un soporte conceptual inestable y muy cuestionado por las cortes en Estados Unidos $^{39}$. Quienes la defienden ${ }^{40}$ aseguran que la interrelación entre las tres categorías ha ayudado a que la aplicación del agotamiento de los DPI sea modulada jurisdiccionalmente en consideración a los efectos que esta aplicación pueda tener sobre la competencia ${ }^{41}$.

35 Christina Bohannan \& Herbert Hovenkamp, Creation without Restraint. Promoting Liberty and Rivalry in Innovation, 365 (Oxford University Press, Oxford, 2012). CARLos ANDRÉs URIBEPiedrahita, Cuestiones concurrenciales y marcarias en la distribución de piezas de recambio (la problemática de los aftermarkets), en Marcas y distribución comercial, 213-268 (EDUARDO Galán-Corona \& Fernando Carbajo-Cascón, coords., Ediciones Universidad de Salamanca, Salamanca, 2011).

36 Yonatan Even, Appropriability, First Sale \& Exhaustion, 1-77, 5 (September 28, 2008). Disponible en: http://ssrn.com/abstract $=1274822$

37 Diego Chijane-Dapkevicius, Derecho de marcas. Función y concepto, nulidades, registro, representación gráfica. Derecho comparado, 357 (1ª ed., Editorial B de F, Montevideo, 2011).

38 Christina Bohannan \& Herbert Hovenkamp, Creation without Restraint. Promoting Liberty and Rivalry in Innovation, 367 (Oxford University Press, Oxford, 2012).

39 Yonatan Even, Appropriability, First Sale \& Exhaustion, 1-77 (September 28, 2008). Disponible en: http://ssrn.com/abstract $=1274822$

40 Otros incluso aseguran que el elemento de competencia ha estado presente durante toda la evolución de la figura en relación con las restricciones posventa al mercado. Her BerT J. HovENKAMP, Innovation and Competition Policy, Ch. 10 (2 ${ }^{\text {nd }}$ ed.): Post-Sale and Related Distribution Restraints Involving IP Rights, Cases and Materials on Innovation and Competition Policy, Chapter 10 (2013). Disponible en: http://papers.ssrn.com/sol3/papers.cfm?abstract_id=1949392. Her bert J. Hovenkamp, IP and Antitrust Policy: A Brief Historical Overview (University of Iowa Legal Studies Research Paper, 05-31, 2005). Disponible en: http://papers.ssrn.com/sol3/ papers.cfm?abstract_id $=869417$

41 Christina Bohannan \& Herbert Hovenkamp, Creation without Restraint. Promoting Liberty and Rivalry in Innovation, 369 (Oxford University Press, Oxford, 2012). GuY A. RuB, Rebal- 
La clara evolución de la relación: derechos de propiedad intelectual-derecho de la competencia, en la aplicación de la figura del agotamiento de los DPI, tuvo un desarrollo homogéneo en la jurisprudencia de Estados Unidos hasta 2008, cuando aparece la sentencia de la Corte Suprema en el caso Quanta Computer, Inc. v. LG Electronics, Inc., $128 \mathrm{~S}$. Ct $2109(2008)^{42}$. En este caso, la Corte Suprema analizó el contenido del contrato de licenciamiento de patente celebrado entre LG Electronics e Intel Corporation para poder concluir si el derecho de propiedad intelectual de LG Electronics se había agotado o no frente a los usos realizados por un comprador de Intel Corporation, esto es, Quanta Computer, Inc. En el contrato de licencia de una patente de procedimiento celebrado entre los dos primeros, para que Intel Corporation pudiera fabricar microprocesadores con esa patente, los cuales iba a comercializar posteriormente, LG Electronics condicionó la licencia a que los compradores de Intel no pudieran utilizar los microprocesadores junto con productos hechos por terceros fabricantes.

En el caso Quanta Computer, la Corte - al reconocer el agotamiento del DPI de LG - rompió con la estrecha relación entre los derechos de propiedad intelectual y el derecho de competencia, construida por la jurisprudencia de Estados Unidos. El fundamento utilizado por la Corte $^{43}$ se ha entendido de dos formas: (i) como un retroceso, pues las restricciones posventa son juzgadas sospechosas ex ante, y los derechos de propiedad intelectual son considerados como inherentemente monopolísticos ${ }^{44}$; o (ii) como la separación de los efectos entre categorías jurídicas distintas, un abandono de la interrelación entre derechos de propiedad intelectual, los contratos y la libre competencia ${ }^{45}$. Es decir, una reafirmación del vínculo ex-

ancing Copyright Exhaustion, 64 Emory Law Journal, 741-817 (2015). Disponible en: http:// law.emory.edu/elj/_documents/volumes/64/3/articles/rub.pdf

42 Estados Unidos, Corte Suprema, Quanta Computer, Inc. v. LG Electronics, Inc., 128 S. Ct 2109 (2008). Disponible en: http://www.supremecourt.gov/opinions/07pdf/06-937.pdf

43 El juez Clarence Thomas, al exponer la decisión unánime de la Corte, simplemente advirtió que "la venta autorizada de un bien en el que se materializa sustancialmente una patente, agota el derecho de patente del titular, e impide que este último invoque el título de patente para controlar la posventa del mismo bien". Estados Unidos, Corte Suprema, Quanta Computer, Inc. v. LG Electronics, Inc., 128 S. Ct 2109 (2008). Disponible en: http://www.supremecourt. gov/opinions/07pdf/06-937.pdf

44 Christina Bohannan \& Her bert Hovenkamp, Creation without Restraint. Promoting Liberty and Rivalry in Innovation, 369 (Oxford University Press, Oxford, 2012).

45 Ariel Katz, What Antitrust Law Can (and Cannot) Teach About the First Sale Doctrine (January 23, 2012). Disponible en: http://papers.ssrn.com/sol3/papers.cfm?abstract_id=1845842 
clusivo de la figura del agotamiento con las teorías de los derechos de propiedad intelectual.

Esta tendencia ha sido reforzada con otra sentencia más reciente: John Wiley \& Sons, Inc. v. Supap Kirtsaeng $(2011)^{46}$, en la que los demandantes John Wiley \& Sons desistieron, durante el proceso, del uso del argumento anticompetitivo; y la Corte Suprema, por su parte, no hizo mención alguna a la interrelación con la libre competencia ${ }^{47}$.

\section{El caso de los procesos de integración en la Unión Europea}

En Europa, encontramos los primeros antecedentes jurisprudenciales justo después del surgimiento de la doctrina en Estados Unidos. En Alemania, las sentencias Kölnisch Wasser y Mariani (ambas de 1902) buscaron evitar el control sobre los canales de distribución que conducen hasta el consumidor final por parte del titular de derechos de propiedad intelectual ${ }^{48}$. Tales antecedentes son la manifestación

46 Estados Unidos, Corte de Apelaciones del 2 Circuito, John Wiley \& Sons, Inc. v. Supap Kirtsaeng (US Court of Appeals for the Second Circuit, $2^{\text {nd }}$ Cir. 2011). Disponible en: http://law. justia.com/cases/federal/appellate-courts/ca2/09-4896/09-4896_complete_opn-2011-08-15. html

47 Esta posición se puede ejemplificar con los comentarios del juez Antonin Scalia de la Corte Suprema de Estados Unidos, quien afirmó en su salvamento de voto en el caso Actavis: "La clave, por supuesto, es que el titular de la patente, cuando realice cualquier acto, incluyendo conciliar, debe actuar dentro del ámbito de la patente. Si su actuación sobrepasa el monopolio de poderes otorgados por la patente, nosotros hemos sostenido que, solo entonces, tales acciones resultan susceptibles al escrutinio del derecho de la competencia" (traducción libre del autor). Estados Unidos, Corte Suprema, Federal Trade Commission v. Actavis, Case No. 12-416 (June 17, 2013). Disponible en: http://www.supremecourt.gov/opinions/12pdf/12-416_m5n0. pdf

48 Juan Ignacio Ruiz-Peris, Agotamiento comunitario de la marca y agotamiento internacional en la doctrina del Tribunal de Justicia, en El papel de la jurisprudencia en el TJCE en la armonización del derecho europeo - situación y perspectiva tras cincuenta años, 353-392 (1ª ed., Salvador Vilata-Menadas, coord., Universitat de València, València, 2005). En la misma línea, Christopher Stothers trae un extracto de la sentencia Guajakol-Karbonat, también de 1902: "The effect of a patent (for a process) is that no-one, except the proprietor (or the persons whom he has authorized) may manufacture a product by the said process and put it on the domestic market. By this act, however, the effect of the protection conferred by the patent is exhausted. The proprietor who has manufactured the product and has put it on the market under this protection which excludes competition from other parties, has enjoyed advantages which the patent confers upon him and has thus exhausted his right". Christopher Stothers, Patent Exhaustion: the UK Perspective, 16 Annual Conference on Intellectual Property Law and Policy, Fordham University School of Law, 27-28 March 2008. Disponible en: http://fordhamipconference.com/wp-content/uploads/2010/08/Christopher-Sothers-Patent-Exhaustion. pdf 
directa de la llamada specific subject matter doctrine ${ }^{49}$, analizada por primera vez en Alemania por Josef Kohler, "quien elaboró el principio de conexión entre los diferentes actos de explotación de la patente" $" 50$. De acuerdo a este principio, los actos de explotación legalmente reconocidos demarcan la extensión del derecho del titular y el límite al provecho económico que este puede obtener; así, el titular del derecho de propiedad intelectual no estará legitimado para ejercer sus facultades cuando un determinado uso esté fuera del alcance del derecho mismo, es decir, el titular habrá agotado sus derechos ${ }^{51}$.

En relación con la integración europea, podemos señalar que en 1971 el Tribunal de Justicia de la entonces Comunidad Económica Europea decidió que el ejercicio del derecho exclusivo de un productor de fonogramas para distribuir sus grabaciones no puede extenderse hasta permitirle prohibir la comercialización de estos productos en un país cuando él mismo ya ha consentido la distribución de las copias en otro país miembro de la Comunidad. Así, en el caso Deutsche Grammophon, GmbH contra Metro-SBGrossmarket, $\mathrm{GmbH} \& \mathrm{Co}^{52}$, teniendo en cuenta las disposiciones sobre libre circulación de mercancías consagradas para la época en el Tratado Constitutivo de la Comunidad Económica Europea ${ }^{53}$, el Tribunal de Justicia concluyó que el ejercicio del derecho exclusivo para distribuir copias de una grabación sonora puede atentar contra el propósito de fusionar los mercados nacionales en un mercado único, en la medida en que el único fundamento para justificar la prohibición de comercialización de los productos en la República Federal de Alemania fue su procedencia desde Francia, en donde

49 Guy Tritton, Intellectual Property in Europe, 648 ( $3^{\text {rd }}$ ed., Sweet \& Maxwell, London, 2008).

50 Antoni Rubi-Puig, Copyright Exhaustion Rationales and Used Software: A Law and Economics Approach to Oracle v. UsedSoft, 4 Journal of Intellectual Property, Information Technology and e-Commerce Law, JIPITEC, 3, 159-178, 162 (2013). Disponible en: http://papers.ssrn.com/sol3/ papers.cfm?abstract_id=2408659

51 Josef Kohler, Handbuch des Deutschen Patentrechts in Rechtsvergleichender Darstellung, 452 ss. (1 $1^{\text {st }}$ ed., Druck und Verlag von J. Bensheimer, Mannheim, 1900).

52 Tribunal de Justicia de las Comunidades Europeas, Deutsche Grammophon, GmbH contra Metro-SB-Grossmarket, GmbH \& Co., asunto 78/70 (1971). Disponible en: http://www.ippt. eu/files/1971/IPPT19710608_ECJ_Deutche_Grammophon_Gesellschaft_v_Metro.pdf

53 Tratado constitutivo de la Comunidad Económica Europea, Tratado CEE, Roma, 25 de marzo de 1957, en especial artículos 3, 5, 36, 86, 86 y 177. Disponible en: http://eur-lex.europa.eu/ legal-content/ES/TXT/?uri=URISERV:xy0023. 
una filial del titular había realizado de forma legítima la primera venta $^{54}$.

Más adelante, en los casos Central Farm contra Winthrop y Central Farm contra Sterling Drug (ambos en 1974), el Tribunal de Justicia terminó de dar forma a la teoría del agotamiento al reconocer que por medio de esta se acotaba el derecho de propiedad intelectual exclusivo hasta "la primera puesta en circulación de los bienes en el mercado comunitario" $" 55$.

Aunque en las sentencias hito antes mencionadas se encontrarán referencias a las doctrinas de la propiedad absoluta y a la specific subject matter doctrine como explicaciones subyacentes del agotamiento del derecho, lo cierto es que tanto en Grammophon como en Central Farm se enfatiza en que la construcción de la figura del agotamiento en la Comunidad Europea se ha hecho sobre la necesidad de garantizar un mercado único (comunitario) y evitar las amenazas a las disposiciones de libre circulación de bienes consagradas en los tratados comunitarios, por ejemplo, para la época el Tratado de Roma de $1957^{56}$.

Una serie de $\operatorname{casos}^{57}$ posteriores a la consolidación de la figura del agotamiento aplicaron de igual forma los artículos 28 y 30 del

54 En este sentido, el Tribunal de Justicia justificó la libre circulación de mercancías (para proteger el mercado único) mediante argumentos procedentes del derecho de la competencia y del derecho contractual, para finalmente poder concluir una regla de propiedad intelectual. GuY Tritton, Intellectual Property in Europe ( $3^{\text {rd }}$ ed., Sweet \& Maxwell, London, 2008). Esto reafirma la autodeterminación amplia que mantienen los países miembros de la Unión Europea en materia de derechos de propiedad, la cual motivó el acercamiento entre la competencia, los contratos y el agotamiento de los DPI. Guido WestKamp, Intellectual Property, Competition Rules and the Emerging Internal Market: Some Thoughts on the European Exhaustion Doctrine, 11 Marquette Intellectual Property Law Review, 2, 291-335 (2007). Disponible en: http:// scholarship.law.marquette.edu/cgi/viewcontent.cgi?article $=1096 \&$ context $=i p l r$

55 El caso Sirena v. Eda es un antecedente de la consolidación que se dio en 1974. Tribunal de Justicia de las Comunidades Europeas, TJCE, Sirena S.r.l. v. Eda S.r.l., caso 40/70, E.C.R. 69 (1971).

56 Guy Tritton, Intellectual Property in Europe, 653, 655 ( $3^{\text {rd }}$ ed., Sweet \& Maxwell, London, 2008).

57 Tribunal de Justicia de las Comunidades Europeas, TJCE, IHT Internationale Heiztechnik GmbH v. Ideal-Standard GmbH, E.C.R. I-2789 (1994). Disponible en: http://curia.europa. eu/juris/liste.jsf?num=C-9/93. Tribunal de Justicia de las Comunidades Europeas, TJCE, Pharmon BV v. Hoechst AG, E.C.R. 2281 (1985). Disponible en: http://www.ippt.eu/files/1985/ IPPT19850709_ECJ_Pharmon.pdf. Tribunal de Justicia de las Comunidades Europeas, TJCE, Keurkoop BV v. Nancy Kean Gift BV, E.C.R. 2853 (1982). Disponible en: http://biicl. org/files/2026_c-144-81.pdf. Tribunal de Justicia de las Comunidades Europeas, TJCE, Merck \& Co. v. Stephar BV, E.C.R. 2063 (1981). http://www.ippt.eu/files/1981/IPPT19810714_ECJ_ Merck_v_Stephar.pdf. Tribunal de Justicia de las Comunidades Europeas, TJCE, MusikVertrieb Membran GmbH v. GEMA, E.C.R. 147 (1981). Disponible en: http://www.ippt.eu/ files/1981/IPPT19810120_ECJ_Membran_and_K-Tel_v_GEMA.pdf 
Tratado de Funcionamiento de la Unión Europea (TFUE ${ }^{58}$ ), lo cual creó la jurisprudencia que permeó la legislación secundaria en Europa y, consecuentemente, las leyes nacionales.

Recientemente, el Tribunal de Justicia de la Unión Europea en el caso UsedSoft GmbH v. Oracle International Corp. (Sentencia de la Corte de Justicia de la Unión Europea del 3 de julio de 2012; C-128/11), tomó la polémica decisión de admitir que la descarga de un software licenciado para un número restringido de usuarios y para el cumplimiento de sus fines propios, da pie para que el derecho de propiedad intelectual del titular, Oracle, se agote; permitiendo consecuentemente que el licenciatario pudiese vender su licencia a un tercero a través de los servicios prestados por UsedSoft ${ }^{59}$.

En UsedSoft v. Oracle, la Corte de Justicia de la UE ratificó el fundamento de la figura del agotamiento de los DPI en Europa, al enfatizar que el propósito de esta figura "es limitar, para evitar la compartimentación de los mercados, las restricciones a la distribución de dichas obras a lo que sea estrictamente necesario para preservar el objeto específico de la propiedad intelectual de que se trate (...)"60 (negrilla fuera del texto). Así reitera los argumentos que llevaron a la consolidación de la figura en el mercado comunitario y que han sido expuestos en la jurisprudencia comunitaria invariablemente, desde Grammophon, GmbH contra Metro-SB-Grossmarket, GmbH \& Co., hasta UsedSoft GmbH v. Oracle International Corp., casos ya mencionados, pero también expuesto en otros casos, como $\mathrm{Me}$ tronome Musik GmbHv. Music Point Hokamp GmbH ${ }^{61}$, Foreningen

58 Unión Europea, Tratado de Funcionamiento de la Unión Europea, TFUE, Roma, 25 de marzo de 1957. Versión consolidada disponible en: http://www.boe.es/buscar/doc.php?id=DOUEZ-2010-70006

59 Para ampliar información sobre el caso UsedSoft, Yin Harn-Lee, UsedSoft GmbH v. Oracle International Corp (Case C-128/11) - Sales of "Used" Software and the Principle of Exhaustion, 43 IIC, International Review of Intellectual Property and Competition Law, 846-853, (2012). Tueerd Overdijk, Polo van der Putt, Eva de Vries \& Thomas Schafft, Exhaustion and Software Resale Rights. A Comparison between the European Exhaustion Doctrine and the U.S. First Sale Doctrine in the Light of Recent Case Law, 2 CRi, 33-39 (2011). Disponible en: http:/l www.vondst-law.com/files/Exhaustion $\% 20$ and $\% 20$ Software $\% 20$ Resale $\% 20$ Rights $\% 20$ CRI $\% 20$ 2011-02.pdf. Paul L. C. Torremans, The Future Implications of the Usedsoft Decision (CREATe Working Paper, 2, 2014). Disponible en: http://zenodo.org/record/8382/files/CREATe-WorkingPaper-2014-02.pdf

60 Tribunal de Justicia de la Unión Europea, TJUE, UsedSoft GmbH v. Oracle International Corp. C-128/11, parágrafo 62 (3 de julio de 2012). Disponible en: http://curia.europa.eu/juris/ document/document.jsf?docid $=124564 \&$ doclang=en

61 Tribunal de Justicia de las Comunidades Europeas, TJCE, Metronome Musik GmbH v. Music Point Hokamp GmbH, C-200/96, Rec. p. I-1953, apartado 14 del 28 de abril de 1998. Disponible 
af danske Videogramdistributører, FDV v. Laserdisken ${ }^{62}$ y Football Association Premier League y otros v. QC Leisure y otros ${ }^{63}$.

A pesar de haber identificado la unificación del mercado interno europeo como la explicación final en la totalidad de las decisiones judiciales que tratan sobre el agotamiento de los DPI, parece que tal justificación para la doctrina por sí sola no es suficiente para entender la aplicación de esta figura. De esta forma, la literatura jurídica recurre frecuentemente a teorías utilitarias ${ }^{64}$ sobre la propiedad intelectual con el fin de explicar el desarrollo del agotamiento de los derechos, bien desde la perspectiva de la subject matter doctrine ${ }^{65}$, o bien desde la teoría de la recompensa ${ }^{66}$.

\section{La teoría inconducente del caso colombiano}

En el caso colombiano, los orígenes del agotamiento de DPI no siguen la misma estructura identificada en Estados Unidos y en la Unión Europea, debido a que el origen de la figura del agotamiento de los DPI no está en el desarrollo jurisprudencial, sino que aparece directamente en las normas que regulan la propiedad intelectual. Igualmente, la jurisprudencia andina carece de ejemplos en los que se haya abordado principalmente algún problema relacionado con el agotamiento del derecho; sí se encuentran referencias al margen con relación a casos en los que se estudian los límites a los derechos de propiedad intelectual o las restricciones a la importación de productos dentro de la Comunidad. En las sentencias del Tribunal de Justicia de la Comunidad Andina en los procesos 2-AI-96 de

en: http://dejure.org/dienste/vernetzung/rechtsprechung?Text=C-200/96

62 Tribunal de Justicia de las Comunidades Europeas, TJCE, Foreningen af danske Videogramdistributorer, FDV v. Laserdisken, C-61/97, Rec. p. I-5171, apartado 13 (22 de septiembre de 1998). Disponible en: http://curia.europa.eu/juris/showPdf.jsf?docid=44104\&doclang=ES

63 Tribunal de Justicia de la Unión Europea, TJUE, Football Association Premier League y otros v. QC Leisure y otros, C-403/08 y C-429/08, Rec. p. I-9083, apartado 106 (4 de octubre de 2011). Disponible en: http://curia.europa.eu/juris/liste.jsf?num=C-403/08\&language $=$ en

64 Antoni Rubi-Puig, Copyright Exhaustion Rationales and Used Software: A Law and Economics Approach to Oracle v. UsedSoft, 4 Journal of Intellectual Property, Information Technology and e-Commerce Law, JIPITEC, 3, 159-178, 162 (2013). Disponible en: http://papers.ssrn.com/sol3/ papers.cfm?abstract_id $=2408659$

65 Guy Tritton, Intellectual Property in Europe ( $3^{\text {rd }}$ ed., Sweet \& Maxwell, London, 2008). ver nota 45 supra

66 Louise Longdin \& Phen Hoon Lim, Inexhaustible Distribution Rights for Copyright Owners and the Foreclosure of Secondary Markets for Software, 44 IIC, International Review of Intellectual Property and Competition Law, 5, 541-568 (2013). 
1996 y 17-IP-98, de $1998^{67}$, esa institución se limita a mencionar la figura del agotamiento explícitamente prevista en la entonces vigente Decisión Andina 344 de $1993^{68}$.

El tercer caso en que el Tribunal Andino abordó el tema del agotamiento es el proceso $24-I P-2005^{69}$, pues al examinar las importaciones paralelas reconoció el agotamiento de los DPI como un límite a estos (siendo este caso un problema del derecho exclusivo sobre una marca). Así, para justificar la legitimidad de las importaciones paralelas al mercado andino, el Tribunal afirmó que el titular de una marca perderá "todos sus derechos" sobre el bien vendido, mientras no se haya alterado la "marca, su distintividad o la calidad del bien"70.

Aunque el Tribunal de Justicia de la Comunidad Andina reconoce el agotamiento de los DPI como un límite a las facultades de los titulares de derechos, tal afirmación se hace desde una justificación proteccionista del mercado y se aleja de la teoría de la recompensa ${ }^{71}$, esta última relacionada con la conexión entre los actos de explotación ${ }^{72}$.

67 Tribunal de Justicia de la Comunidad Andina, Proceso 2-AI-96 (20 de junio de 1997). Disponible en: http://intranet.comunidadandina.org/Documentos/DocumentosEntrada/ E00000622011.pdf. Tribunal de Justicia de la Comunidad Andina, Proceso 17-IP-98 (21 de abril de 1998). Disponible en: http://intranet.comunidadandina.org/documentos/DocumentosEntrada/E00012222011.pdf

68 Esta figura ya estaba prevista desde la Decisión 311 de 1991, en su artículo 98. Comunidad Andina, Decisión Andina 311, que establece el Régimen Común sobre Propiedad Industrial (8 de noviembre de 1991). Disponible en: http://www.wipo.int/wipolex/es/text.jsp?file_id=224231. Comunidad Andina, Decisión Andina 344, que establece el Régimen Común sobre Propiedad Industrial (21 de octubre de 1993). Disponible en: http://www.wipo.int/wipolex/es/details. jsp?id=9454

69 Tribunal de Justicia de la Comunidad Andina, Proceso 24-IP-2005 (25 de mayo de 2005). Disponible en: http://intranet.comunidadandina.org/documentos/DocumentosEntrada/ E00015142011.pdf

70 Tal requisito, presente en el derecho de marcas de Estados Unidos, la Unión Europea y la normatividad andina, refuerza el punto de quienes consideran que el agotamiento recae sobre derechos y no sobre el objeto que los incorpora. En este sentido, GUY TRITTON, Intellectual Property in Europe, 653, 655 ( $3^{\text {rd }}$ ed., Sweet \& Maxwell, London, 2008).

71 "The patentee or his assignee having in the act of sale received all the royalty or consideration which he claims for the use of his invention in that particular machine or instrument", Estados Unidos, Corte Suprema, Adams v. Burke, 84 U.S. 453 (1873). Disponible en: https://supreme. justia.com/cases/federal/us/84/453/case.html. PETER JAsZI, Toward a Theory of Copyright: The Metamorphoses of 'Authorship', 1991 Duke Law Journal, 455-502, 478 (1991). Disponible en: http://scholarship.law.duke.edu/cgi/viewcontent.cgi?article=3150\&context=dlj. WILLIAM W. FISHER, III, The Growth of Intellectual Property: A History of the Ownership of Ideas in the United States/Geistiges Eigentum - ein ausufernder Rechtsbereich: Die Geschichte des Ideenschutzes in den Vereinigten Staaten, Eigentum im Internationalen Vergleich, 265-291 (Vandenhoeck \& Ruprecht, 1999). Disponible en: http://cyber.law.harvard.edu/people/tfisher/iphistory.pdf

72 José Massaguer-Fuentes, El contenido y alcance del derecho de patente, Actualidad Jurídica 
La normativa andina reconocía ${ }^{73}$ la posibilidad de coexistencia de marcas similares de diferentes titulares bajo ciertas condiciones de no uso de una de las marcas en un territorio; esta posibilidad se eliminó del ordenamiento andino en 1991 cuando la Decisión Andina 311 reconoció por primera vez el agotamiento del derecho. En su momento, el Tribunal Andino afirmó que: "La Decisión 311 que sustituyó la Decisión 85 optó más bien por privilegiar la protección marcaria legítimamente adquirida en el territorio de un País Miembro y no permitir la coexistencia, cuando derogó el artículo 75 citado, limitándose a establecer en su lugar, el principio del agotamiento del derecho"74.

Teniendo en cuenta que en el mismo pronunciamiento antes señalado, el Tribunal se refirió a la coexistencia como una medida que "claramente optaba por privilegiar el libre comercio" y la equipara a la figura del agotamiento del derecho, el agotamiento de los DPI dentro del ordenamiento andino es una herramienta con un propósito de protección del mercado, en el cual el análisis sobre el alcance de los derechos de propiedad intelectual interesa en la medida de los efectos que estos producen o no en el mercado andino ${ }^{75}$.

Antes de la consagración en el ordenamiento andino, no se pueden ubicar antecedentes normativos o jurisprudenciales sobre el agotamiento de los DPI en Colombia; tampoco en la jurisprudencia o leyes internas aplicables en el país. De forma que no ha sido posible identificar las teorías que explican la figura del agotamiento de los DPI en Colombia. Con los escasos recursos disponibles,

Uría Menéndez, Homenaje al profesor D. Rodrigo Uría González en el centenario de su nacimiento, número extraordinario, 173-187 (2006). Disponible en: http://www.uria.com/documentos/ publicaciones/1632/documento/art12.pdf?id=2158

73 En las decisiones andinas 85 y 344, antecesoras de la actual 486. Comunidad Andina, Decisión Andina 85, que establece el Reglamento para la Aplicación de las Normas sobre Propiedad Industrial (5 de junio de 1974). Disponible en: http://www.wipo.int/wipolex/es/ details.jsp?id=9464. . Comunidad Andina, Decisión Andina 344, que establece el Régimen Común sobre Propiedad Industrial (21 de octubre de 1993). Disponible en: http://www.wipo. int/wipolex/es/details.jsp?id=9454. Comunidad Andina, Decisión Andina 486, que establece el Régimen Común sobre Propiedad Industrial (14 de septiembre de 2000). Disponible en: http://www.wipo.int/edocs/lexdocs/laws/es/can/can012es.pdf

74 Tribunal de Justicia de la Comunidad Andina, Proceso 2-AI-96, 48 (20 de junio de 1997). Disponible en: http://intranet.comunidadandina.org/Documentos/DocumentosEntrada/ E00000622011.pdf

75 Carolina Barragán, Miguel Ceballos, Diana Marín \& Óscar Tamayo, Agotamiento del derecho a la luz del derecho comunitario. Unión Europea y Comunidad Andina, 16 Revista La Propiedad Inmaterial, 225-280, 266 (2012). Disponible en: http://revistas.uexternado.edu.co/ index.php/propin/article/view/3271/3680 
únicamente se puede enlazar esta figura al proceso de integración económica y de mercados que tiene como propósito la Comunidad Andina de Naciones.

No obstante lo anterior, Mauricio Velandia identifica al agotamiento de los DPI con el interés general protegido mediante las prohibiciones de: “(i) acuerdos verticales (precio, producto y territorio) ${ }^{76}$; (ii) abuso de la posición de dominio (imposición de doble margen, con ganancia para fabricante y distribuidor), $\mathrm{y}$ (iii) competencia desleal (afectación a la concurrencia)" ${ }^{\text {"77. De esta }}$ forma, la explicación propuesta por Velandia (aunque no apunta hacia el origen de la figura en Colombia) asocia al agotamiento de los DPI como una herramienta para asegurar la libre competencia dentro del mercado (andino) $)^{78}$.

\section{4. ¿El agotamiento del derecho: una figura de origen lo- cal o internacional?}

Aunque el análisis de la relación entre el agotamiento de los DPI y el derecho internacional se separa del origen jurisprudencial de la figura, hoy no se puede abordar ningún aspecto de la propiedad intelectual sin remitir la discusión al plano de los tratados internacionales, que contienen la regulación mínima sobre el alcance de la figura del agotamiento de los DPI que deben asegurar sus países firmantes. Sin embargo, más allá de los muy significativos

76 Aunque debe apuntarse que en Colombia los acuerdos verticales no resultan en afectaciones a la libre competencia per se.

77 Mauricio Velandia, Agotamiento del derecho de marca y su relación con el derecho de la competencia, en Derecho internacional de los negocios. Alcances, 427-442 (1 ${ }^{\text {a }}$ ed., Adriana Zapata de Arbeláez, comp., Universidad Externado de Colombia, Bogotá, 2009).

78 Tribunal de Justicia de las Comunidades Europeas, TJCE, IHT Internationale Heiztechnik GmbH v. Ideal-Standard GmbH, E.C.R. I-2789 (1994). Disponible en: http://curia.europa. eu/juris/liste.jsf?num=C-9/93. Tribunal de Justicia de las Comunidades Europeas, TJCE, Pharmon BV v. Hoechst AG, E.C.R. 2281 (1985). Disponible en: http://www.ippt.eu/files/1985/ IPPT19850709_ECJ_Pharmon.pdf. Tribunal de Justicia de las Comunidades Europeas, TJCE, Keurkoop BV v. Nancy Kean Gift BV, E.C.R. 2853 (1982). Disponible en: http://biicl. org/files/2026_c-144-81.pdf. Tribunal de Justicia de las Comunidades Europeas, TJCE, Merck \& Co. v. Stephar BV, E.C.R. 2063 (1981). http://www.ippt.eu/files/1981/IPPT19810714_ECJ_ Merck_v_Stephar.pdf. Tribunal de Justicia de las Comunidades Europeas, TJCE, MusikVertrieb Membran GmbH v. GEMA, E.C.R. 147 (1981). Disponible en: http://www.ippt.eu/ files/1981/IPPT19810120_ECJ_Membran_and_K-Tel_v_GEMA.pdf. Antoni Rubí-Puig, Copyright Exhaustion Rationales and Used Software: A Law and Economics Approach to Oracle v. UsedSoft, 4 Journal of Intellectual Property, Information Technology and e-Commerce Law, JIPITEC, 3, 159-178, 162 (2013). Disponible en: http://papers.ssrn.com/sol3/papers. cfm?abstract_id=2408659 
Convenios de París (1883) y de Berna (1886), en los que no hay referencias a la figura del agotamiento de los DPI, solamente en 1994, con la aparición de los ADPIC, se concreta el primer instrumento internacional que trata explícitamente a los bienes protegidos por la propiedad intelectual como objetos de comercio internacional, cuya regulación puede incidir de forma importante en la "reducción de las distorsiones del comercio internacional y los obstáculos al mismo"79.

En este tratado, el agotamiento del derecho se encuentra en el artículo sexto, que expresamente dispuso: "no se hará uso de ninguna disposición del presente Acuerdo en relación con la cuestión del agotamiento de los derechos de propiedad intelectual", dejando en libertad ${ }^{80}$ a los países miembros para regular internamente esta materia $^{81}$. Aunque algunos consideran que el tratado de los ADPIC permite cierta flexibilidad ${ }^{82}$ en la regulación interna de la figura del agotamiento del derecho, la misma no podría contravenir el "espíritu del Acuerdo", en especial cuando se habla del agotamiento internacional de los $\mathrm{DPI}^{83}$. Tal flexibilidad restringida no solo se ha señalado respecto de los máximos del agotamiento, sino también para los mínimos, es decir, cuando los tratados internacionales dejan en libertad a los Estados firmantes para regular el agotamiento de los DPI, a su vez estarían imponiendo como mínimo el agota-

79 Organización Mundial del Comercio, OMC, Acuerdo sobre los Aspectos de los Derechos de Propiedad Intelectual relacionados con el Comercio (Acuerdo sobre los ADPIC) o TradeRelated aspects of Intellectual Property Rights, TRIPS, Anexo 1C del Convenio por el que se crea la OMC, preámbulo, firmado en 1994. Disponible en: https://www.wto.org/spanish/ docs_s/legal_s/legal_s.htm https://www.wto.org/spanish/docs_s/legal_s/27-trips.pdf

80 Hanns Ullrich, TRIPS: Adequate Protection, Inadequate Trade, Adequate Competition Policy, 4 Pacific Rim Law \& Policy Journal, 1, 153-210, 191 (1995). Disponible en: http:// digital.law.washington.edu/dspace-law/bitstream/handle/1773.1/933/4PacRimLPolyJ153. pdf?sequence $=1 \&$ isAllowed $=y$

81 Interpretación reconocida posteriormente en la Declaración de Doha. Organización Mundial del Comercio, OMC, Declaración Ministerial de Doha relativa al acuerdo sobre los ADPIC y la salud pública, literal d del numeral 5 (2001), Doha, Catar, 14 de noviembre de 2001. Disponible en: https://www.wto.org/spanish/thewto_s/minist_s/min01_s/mindecl_s.htm

82 Alexander B. Pope, Note: A Second Look at First Sale: An International Look at U.S. Copyright Exhaustion, 19 Journal of Intellectual Property Law, 201-230 (2011).

83 José Massaguer-Fuentes, El agotamiento del derecho de marca y las importaciones paralelas, 5 Temas de Derecho Industrial y de la Competencia, 285-328 (2001), cita a THомAs CotTIER, The Prospects for Intellectual Property in GATT, 28 Common Market Law Review, 2, 383-414 (1991). 
miento más reducido ${ }^{84}$, aplicable solo para ciertas eventualidades y solo en el ámbito nacional ${ }^{85}$.

Aunque se pueda tener una fuerte sensación sobre la relevancia internacional de la figura del agotamiento de los $\mathrm{DPI}^{86}$, debido a su impacto en el intercambio global de bienes y servicios, lo cierto es que en los tratados internacionales esta figura no ha sido regulada, o se le ha otorgado una relativa libertad al derecho interno para implementarla (o no) de acuerdo a sus intereses sociales, políticos y/o económicos ${ }^{87}$. En este sentido, el agotamiento del derecho abandona el escenario de política internacional para aterrizar en el campo de las políticas locales sobre participación en el comercio internacional. Es decir, el agotamiento de DPI no se caracteriza por tener una relación internacional $\nabla$ local; sino que obedece a una

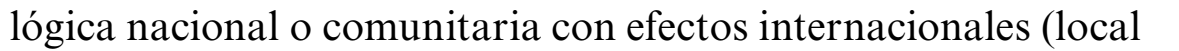
internacional $)^{88}$.

No obstante la aparente libertad local para regular esta figura, las regulaciones en los referentes geográficos estudiados en este trabajo (entendidas como locales en este contexto), comparten una estructura básica de la figura del agotamiento de los DPI y los mismos interrogantes sobre su evolución ${ }^{89}$.

84 En el caso colombiano, el agotamiento de los derechos de autor no está regulado y la imposición de un estándar mínimo implicaría que por lo menos debería aplicarse el agotamiento nacional para el derecho de distribución.

85 Carolina Barragán, Miguel Ceballos, Diana Marín \& Óscar Tamayo, Agotamiento del derecho a la luz del derecho comunitario. Unión Europea y Comunidad Andina, 16 Revista La Propiedad Inmaterial, 225-280, 230 (2012). Disponible en: http://revistas.uexternado.edu.co/ index.php/propin/article/view/3271/3680

86 JuAN DAVID CASTRO-GARCÍA, El agotamiento de los derechos de propiedad intelectual, 13 Revista La Propiedad Inmaterial, 253-282, 255 (2009). Disponible en: http://revistas.uexternado.edu. co/index.php/propin/article/view/463/3631

87 La libertad relativa, en lo mencionado anteriormente. Por ejemplo, en la redacción de los Tratados OMPI sobre Derecho de Autor se limita la figura del agotamiento del derecho a su aplicación: (i) solo en el derecho de distribución; y se afirma que (ii) los países podrían encontrar que no existen las "condiciones necesarias" para regular el agotamiento del derecho. Organización Mundial de Propiedad Intelectual, Tratado de la OMPI sobre Derecho de Autor (WIPO Copyright Treaty, WCT), Ginebra, 20 de diciembre de 1996, artículo 6. Disponible en: http://www.wipo.int/treaties/es/text.jsp?file_id=295167

88 De esta forma, visto desde la perspectiva de derecho internacional, en principio, cada país tiene la libertad de regular el agotamiento de acuerdo a sus propios intereses; esta situación se puede evidenciar en el caso colombiano por la falta de regulación en materia de derecho de autor y las consecuentes interpretaciones alrededor de esta figura. LuIS ÁNGEL MADRID, Importaciones paralelas. Agotamiento del derecho de propiedad intelectual (Universidad Sergio Arboleda, octubre 2005). Disponible en: http://www.usergioarboleda.edu.co/tlc/tlc_importaciones $\% 20$ paralelas.htm

89 Como ya se ha mencionado anteriormente, el caso colombiano es la excepción debido a la escasa conflictividad judicial en la materia. 


\section{SEGUNDA PARTE}

En la primera parte de este trabajo se evaluó el desarrollo de la figura del agotamiento de los DPI, desde sus orígenes hasta los más recientes conflictos sobre propiedad intelectual. Este análisis es presentado desde la perspectiva de lo que Robert P. Merges ha denominado Midlevel Principles, es decir, aquellos principios integradores de teorías más generales (sobre la propiedad intelectual o la propiedad ordinaria) que, aunque continúan siendo conceptos básicos, ayudan a dar sentido a doctrinas, reglas y prácticas en un área del derecho ${ }^{90}$. En la segunda parte, el objetivo será el de retomar las principales teorías identificadas en la primera parte, para luego analizar los efectos de estos marcos teóricos en relación con las tensiones que sufre el agotamiento hoy.

\section{A. Las teorías detrás del agotamiento}

El lector ha podido identificar en el apartado anterior dos grupos de explicaciones prevalentes a la hora de justificar al agotamiento: las explicaciones propietarias ${ }^{91}$ y las explicaciones de mercado. También se ha hablado sobre el ordenamiento internacional como una explicación de la figura, al haberse podido comprobar que la existencia del agotamiento está sustentada en las políticas locales sobre participación en los mercados internacionales; esto último a pesar de la expresa consagración en tratados internacionales.

Con base en lo anterior, ahora se pretende hacer evidentes las relaciones entre las regulaciones del agotamiento y las justificaciones que las han soportado en su momento, y los efectos derivados del marco teórico utilizado detrás de la misma figura.

90 Robert P. Merges, Justifying Intellectual Property, 139 (Harvard University Press, Cambridge, London, 2011).

91 Aquellas explicaciones que reafirman la equivalencia entre la propiedad intelectual como un derecho de propiedad, una propiedad especial o por lo menos como una relación jurídica real. 


\section{El juego de las justificaciones}

La figura del agotamiento ha sido consagrada normativamente como un límite intrínseco ${ }^{92}$ al derecho de propiedad intelectual ${ }^{93}$ como una consecuencia de la explicación propietaria que justifica su existencia. En este sentido, el agotamiento es reconocido como la frontera hasta donde llega el derecho exclusivo de propiedad intelectual, bien sea porque "naturalmente" el derecho subjetivo es así ${ }^{94}$, o porque el titular del derecho no merece más protección de la que ya se le ha otorgado en relación con su esfuerzo ${ }^{95}$. De otra parte, la figura del agotamiento también ha sido utilizada como un instrumento para la regulación de mercados, tanto en su faceta de política pública de organización de $\operatorname{mercados}^{96}$ (objetivo fijado ex ante), como en el análisis de los efectos anticompetitivos derivados de su aplicación ${ }^{97}$ (ponderación ex post); situación que por supuesto es resultado de una explicación de mercados sobre la figura del agotamiento de los DPI.

Como se ha descrito en el apartado anterior del presente artículo, estas dos explicaciones no se excluyen mutuamente, al punto que hoy se encuentran explicaciones conjuntas sobre del agotamiento ${ }^{98}$;

92 Yonatan Even, Appropriability, First Sale \& Exhaustion, 1-77 (September 28, 2008). Disponible en: http://ssrn.com/abstract=1274822. Casos como Estados Unidos, Corte de Apelaciones del 8 Circuito, National Phonograph Co. v. Schlegel, 128 F. 733 (US Court of Appeals for the Eighth Circuit, $8^{\text {th }}$ Cir. 1904) y Estados Unidos, Corte Suprema, Bobbs-Merrill Co. v. Straus, 201 U.S. 339 (1908). Disponible en: http://caselaw.findlaw.com/us-supreme-court/210/339.html

93 Estados Unidos, Copyright Act, 17 USCS Sects. 101-810, 1976, art. 109. Disponible en: http:// www.copyright.gov/title17/, http://copyright.gov/title17/92appa.pdf, https://www.law.cornell. edu/copyright/copyright.table.html

94 Antoni Rubi-Puig, Copyright Exhaustion Rationales and Used Software: A Law and Economics Approach to Oracle v. UsedSoft, 4 Journal of Intellectual Property, Information Technology and e-Commerce Law, JIPITEC, 3, 159-178, 162 (2013). Disponible en: http://papers.ssrn.com/sol3/ papers.cfm?abstract_id $=2408659$

95 Se entiende como aquella limitación que tiene su fundamento en la misma finalidad del derecho; en este caso, derecho de propiedad intelectual. Esta se contrapone a una limitación extrínseca. ERnesto Peña-Quiñonez, El derecho de bienes $\left(1^{\text {a }}\right.$ ed., Librería Jurídicas Wilches, Bogotá, 1995). Disponible en: http://www.dasumo.com/libros/el-derecho-de-bienes- $\%$ E2\%80\%93ernesto-pe $\% \mathrm{C} 3 \% \mathrm{~B} 1 \mathrm{a}-\mathrm{qui} \% \mathrm{C} 3 \% \mathrm{~B}$ lonez-pdf.html. Sobre el agotamiento entendido como un límite intrínseco de los DPI, FERNANDo CARBAJO-CASCÓN, La distribución selectiva y el comercio paralelo de productos de lujo ( $1^{\mathrm{a}}$ ed., Grupo Editorial Ibáñez, Bogotá, 2010).

96 Guy Tritton, Intellectual Property in Europe, 653, 655 ( $3^{\text {rd }}$ ed., Sweet \& Maxwell, London, 2008).

97 Melissa Goldberg, A Textbook Dilemma: Should the First Sale Doctrine Provide a Valid Defense for Foreign-Made Goods?, 81 Fordham Law Review, 101-136 (2012). Disponible en: http:// ssrn.com/abstract $=2042811$

98 Louise Longdin \& Phen Hoon Lim, Inexhaustible Distribution Rights for Copyright Owners and the Foreclosure of Secondary Markets for Software, 44 IIC, International Review of Intellectual 
más aún, siempre que pueda ser identificada una explicación de mercado, cualquier que ella sea, al mismo tiempo se podrá encontrar una explicación de propiedad, en la medida en que la figura del agotamiento no ha sido reconocida para todas las facultades del titular de los DPI, ni para todos los tipos de soportes en los que se pueda materializar un objeto protegido por la propiedad intelectual (no es un principio general del sistema de la propiedad intelectual pues se sigue manejando como una excepción). Un ejemplo de tal afirmación son los comentarios del juez Antonin Scalia de la Corte Suprema de Justicia de Estados Unidos en el caso Actavis: "La clave, por supuesto, es que el titular de la patente, cuando realice cualquier acto, incluyendo conciliar, debe actuar dentro del ámbito de la patente. Si su actuación sobrepasa el monopolio de poderes otorgados por la patente, nosotros hemos sostenido que, solo entonces, tales acciones resultan susceptibles al escrutinio del derecho de la competencia" $"$.

\section{Las teorias frente a las tensiones del agotamiento}

En la medida en que las dos explicaciones del agotamiento antes descritas han convivido a lo largo del desarrollo de la figura (el ordenamiento internacional se descarta como una explicación originaria de la figura, de acuerdo a lo explicado anteriormente), las más importantes tensiones del agotamiento pueden ser entendidas desde esas explicaciones, su enfrentamiento, asociación o mimetismo.

El objeto del agotamiento: en primer lugar, quiero comenzar el análisis del objeto del agotamiento con un concepto que se ha incorporado y convive pacíficamente en la regulación, la jurisprudencia y la doctrina; esto es, la distinción entre corpus mechanicum, mero vehículo sensible de la creación, y corpus mysticum, entidad que trasciende toda ejemplarización ${ }^{100}$.

El derecho de propiedad intelectual, como disciplina, se diferencia de la propiedad ordinaria en la medida en que la segunda parte del supuesto de la imposibilidad del disfrute múltiple y simultáneo,

Property and Competition Law, 5, 541-568 (2013).

99 Guy Tritton, Intellectual Property in Europe, 648 ( ${ }^{\text {rd }}$ ed., Sweet \& Maxwell, London, 2008). 100 Tal distinción es generalmente reconocida, aunque algunas teorías puedan tratar de equiparar los efectos. Hermenegildo Baylos-Corroza, Tratado de derecho industrial, 136 ( $3^{\mathrm{a}}$ ed., Civitas, Navarra, 2009). 
goce con el que ha de contar necesariamente la primera discipli$\mathrm{na}^{101}$. Así, el derecho de propiedad intelectual recae sobre el bien intelectual, el corpus mysticum y no sobre el mechanicum ${ }^{102}$.

En consecuencia, cuando nos referimos al agotamiento de los derechos de propiedad intelectual - ya no sobre el derecho de propiedad como tal-, centramos el objeto de estudio en las facultades derivadas de tal disciplina y no sobre las que garantizan la propiedad ordinaria respecto del corpus mechanicum. No obstante constituir un lugar común en la doctrina, e incluso configurar una descripción obvia de la denominación utilizada ${ }^{103}$ (agotamiento de los derechos de propiedad intelectual), afirmar que la figura del agotamiento recae sobre derechos y no sobre los objetos que incorporan el derecho, no es una afirmación plenamente aceptada o un hecho fácilmente comprobable.

En este sentido se han pronunciado Christina Bohannan y Herbert Hovenkamp, al afirmar que una característica esencial del agotamiento (regla de la primera venta) es que esta aplica a las cosas y no a los derechos de propiedad intelectual en abstracto $^{104}$. Esta posición se ha visto sustentada en diferentes asuntos en materia de agotamiento, en especial, en el caso de las licencias de software ${ }^{105}$. En esta concepción, el agotamiento resulta inaplica-

101 Hermenegildo Baylos-Corroza, Tratado de derecho industrial, 136 ( $3^{\mathrm{a}}$ ed., Civitas, Navarra, 2009).

102 Josef Kohler, Handbuch des Deutschen Patentrechts in Rechtsvergleichender Darstellung, 83 ss. ( $1^{\text {st }}$ ed., Druck und Verlag von J. Bensheimer, Mannheim, 1900). En este sentido, se pueden consultar las sentencias que resuelven los casos en que se ratifica la no protección de las meras ideas y la necesidad de expresión en un soporte material para configurar el objeto del derecho de autor, como la sentencia que resolvió la demanda de Luis Alejandro Velasco contra Gabriel García Márquez, por protección al derecho de autor en la novela Relato de un náufrago (Tribunal Superior de Bogotá, Sala Civil, Luis Alejandro Velasco contra Gabriel García Márquez, 27 de enero de 1994, magistrado ponente Ricardo Zopó-Méndez). O la que resolvió las demandas contra el libro El Código Da Vinci. Inglaterra y Gales, Corte de Apelaciones de Londres, División Civil, Michael Baigent y Richard Leigh contra The Random House Group Limited [2007] EWCA Civ 247, Caso A3 2006/0971, Código Da Vinci, jueces John Mummery, Bernard Rix y Anthony Lloyd. Disponible en: http://www.bailii.org/ew/cases/ EWCA/Civ/2007/247.html

103 El agotamiento entendido como un límite a los derechos de propiedad intelectual. JUAN DAVID CAStro-Garcí, El agotamiento de los derechos de propiedad intelectual, 13 Revista La Propiedad Inmaterial, 253-282, 254 (2009). Disponible en: http://revistas.uexternado.edu.co/index.php/ propin/article/view/463/3631

104 Christina Bohannan \& Herbert Hovenkamp, Creation without Restraint. Promoting Liberty and Rivalry in Innovation, 371 (Oxford University Press, Oxford, 2012).

105 Christina Bohannan, Copyright Preemption of Contracts, 67 Maryland Law Review, 611-663 (2008). Disponible en: http://papers.ssrn.com/sol3/papers.cfm?abstract_id=1138251 
ble para las disposiciones hechas bajo licencia ${ }^{106}$, en la medida en que con esta modalidad contractual el titular de los derechos de propiedad intelectual no está distribuyendo (acto entendido como una disposición del bien físico) ${ }^{107}$; y más bien se está restringiendo la disponibilidad de los productos, al tiempo que se está dando la "apariencia" de propiedad al licenciatario ${ }^{108}$.

En el caso de las licencias, suele afirmarse que con el acto de licenciamiento no se está realizando una "primera venta", sino una simple autorización temporal; es decir, no hay una disposición del derecho sino más bien una limitación obligacional; de forma que el titular de los derechos mantendría una potestad decisoria sobre los usos de los bienes que materializan sus DPI. También se ha explicado desde la teoría de la recompensa antes mencionada, pues afirma que el precio de la licencia (menor que el de una cesión de derechos) no incorpora el valor de una transferencia y, en consecuencia, el titular no ha recibido toda la protección que merece del derecho ${ }^{109}$ y deja espacio para que este pueda controlar las copias entregadas bajo licencia. En definitiva, las licencias a diferencia de una trasferencia del dominio, no permiten, en principio, apropiar todos los recursos que se esperarían de una venta.

106 En relación con el caso Capitol Records, LLC v. ReDigi, Inc.: Theodore Serra, Rebalancing at Resale: ReDigi, Royalties, and The Digital Secondary Market, 93 Boston University Law Review, 5, 1753-1801 (2013). Disponible en: http://www.bu.edu/bulawreview/files/2013/10/ SERRA_Resale.pdf. Estados Unidos, Corte del Distrito Sur de New York, Capitol Records, LLC v. ReDigi, Inc., No. 12-95, 2013 WL 1286134 (United States District Court, Southern District of New York, SDNY, March 30, 2013). Disponible en: https://cases.justia.com/federal/ district-courts/new-york/nysdce/1:2012cv00095/390216/109/0.pdf

107 Entre otras sentencias sobre esta misma línea: Estados Unidos, Corte del Distrito Norte de California, División San José, Adobe Systems, Inc. v. Stargate Software Inc., 216 F.Supp.2d 1051 (United States District Court, N.D. California, San Jose Division, N.D. Cal. 2002). Disponible en: https://h2o.law.harvard.edu/cases/4333. Estados Unidos, Corte de Apelaciones del Circuito Federal, DSC Communications Corporation v. Pulse Communications, Inc., 170 F.3d 1354 (United States Court of Appeals, Federal Circuit, Fed. Cir. 1999). Disponible en: http://openjurist.org/170/f3d/1354. Estados Unidos, Corte de Apelaciones del 9 Circuito, MAI Systems Corporation v. Peak Computer, Inc., 991 F.2d 511 (US Court of Appeals for the Ninth Circuit, $9^{\text {th }}$ Cir. 1993). Disponible en: https://www.law.cornell.edu/copyright/cases/991_F2d_511.htm

108 Aaron K. Perzanowski \& Jason Schultz, Digital Exhaustion, 58 University of California, Los Angeles, UCLA Law Review, 889-946, 903 (2011). Disponible en: http://www.uclalawreview. org/pdf/58-4-1.pdf

109 Antoni Rubí-Puig, Copyright Exhaustion Rationales and Used Software: A Law and Economics Approach to Oracle v. UsedSoft, 4 Journal of Intellectual Property, Information Technology and e-Commerce Law, JIPITEC, 3, 159-178, 168 (2013). Disponible en: http://papers.ssrn.com/sol3/ papers.cfm?abstract_id $=2408659$ 
Cualquiera de las dos explicaciones sobre la inoperancia del agotamiento llevaría a confirmar la anterior afirmación de Bohannan y Hovenkamp: el agotamiento no aplica a los DPI en abstracto, sino a los bienes que materializan esos DPI (que circulan en el mercado e interesan al análisis de políticas de competencia o integración), en la medida en que el titular, por medio de la licencia, mantiene su derecho y así no se podría agotar (posición que mantiene el statu quo del titular del derecho subjetivo, en relación con una explicación desde la propiedad) .

Tanto en Estados Unidos, como en la Unión Europea, podemos identificar casos en los que, a pesar de mediar contratos de licencia, los jueces encontraron méritos suficientes para aplicar el agotamiento de los DPI; esto permitió a los licenciatarios disponer del bien en contra de las condiciones de la licencia otorgada. En Estados Unidos, el referente es el caso Quanta antes reseñado, y en la Unión Europea es el caso UsedSoft GmbH v. Oracle International Corp. ${ }^{110}$.

Al habilitar el agotamiento en virtud a una licencia que tiene por objeto permitir el uso de derechos de propiedad intelectual, estos dos antecedentes judiciales parecen retomar el lugar común de la figura; es decir, el agotamiento recae sobre los derechos de propiedad intelectual ${ }^{111}$, lo que se aleja de la aguda pero minoritaria interpretación que hacen Bohannan y Hovenkamp ${ }^{112}$.

110 Reto M. Hilty, Kaya Köklü \& Fabian Hafenbrädl, Software Agreements: Stocktaking and Outlook - Lessons from the UsedSoft v. Oracle Case from a Comparative Law Perspective, 44 International Review of Intellectual Property and Competition Law, 3, 263-292 (2013).

111 Keith Harris, For Promotional Use Only: Is the Resale of a Promotional CD Protected by the First Sale Doctrine?, 30 Cardozo Law Review, 4, 1745-1772 (2009). Disponible en: http://www. cardozolawreview.com/Joomla1.5/content/30-4/HARRIS.30-4.pdf

112 Se califica de minoritaria la afirmación de que el agotamiento recae sobre los objetos que materializan el derecho y no sobre los derechos mismos, toda vez que a este resultado —es decir, la imposibilidad de aplicación del agotamiento para los casos de licencias de usotambién han llegado quienes afirman que el agotamiento recae sobre los derechos de propiedad intelectual. En este sentido, se pueden ver los casos Vernor v. Autodesk o MDY Industries v. Blizzard Entertainment, en los que se reconoce la facultad al titular del software licenciado de restringir a sus usuarios cualquier transferencia del mismo. Estados Unidos, Corte de Apelaciones del 9 Circuito, Vernor v. Autodesk, No. 09-35969 (US Court of Appeals for the Ninth Circuit, 10 September 2010). Disponible en: http://cdn.ca9.uscourts.gov/ datastore/opinions/2010/09/10/09-35969.pdf. Estados Unidos, Corte de Apelaciones del 9 Circuito, MDY Industries v. Blizzard Entertainment, No. 09-15932 (US Court of Appeals for the Ninth Circuit, 17 February, 2011). Disponible en: http://cdn.ca9.uscourts.gov/datastore/ opinions/2011/02/17/09-15932.pdf 
Derechos que se agotan: retomando lo que se ha descrito anteriormente como el lugar común del agotamiento y entendiendo que esta figura aplicará para los derechos de propiedad intelectual, resultará relevante conocer qué derechos se agotan y si no todos los derechos de propiedad intelectual se pueden agotar.

A diferencia de lo que ocurre en el derecho de autor, para el resto de los subsistemas de propiedad intelectual el contenido del derecho protegido es listado por medio del ius prohibendi ${ }^{113}$, mediante el cual el titular de derechos tiene la facultad de excluir a terceros del uso del signo distintivo o la invención protegida ${ }^{114}$. En las regulaciones sobre derecho de autor, la construcción de la protección se hace integrando más estrechamente el ius prohibendi, característico de la propiedad industrial, con el ius utendi $i^{115}$; esta integración se evidencia en la lista, más o menos homogénea, de facultades o derechos patrimoniales de uso que ostenta el autor para explotar su obra. Desde la perspectiva "negativa"116 que caracteriza a la construcción de la propiedad industrial, encontramos también una lista, ya no de facultades para el uso, sino de usos prohibidos o que el titular podrá impedir a terceros. De allí que para analizar qué derecho o facultades se agotan, primero se deba diferenciar entre el agotamiento de la propiedad industrial y el que opera en el derecho de autor.

El primer escollo que se ha presentado al momento de listar los derechos que se agotan ha sido la ausencia de una lista de facultades en materia de propiedad industrial. Tal dificultad se ha superado por medio de una ficción, mediante la cual se les atribuye a las invenciones, signos distintivos y diseños industriales un derecho de

113 El ius prohibendi en materia de propiedad intelectual se diferencia de la facultad de prohibir de la propiedad común, en la medida en que en esta última esa facultad se satisface mediante el aspecto positivo de poseer; mientras que en el caso de la propiedad intelectual el ius prohibendi es un recurso artificial, un verdadero derecho subjetivo que permitirá la exclusión de los demás. Hermenegildo Baylos-Corroza, Tratado de derecho industrial, 283 ( $3^{\text {a }}$ ed., Civitas, Navarra, 2009).

114 J. Thomas McCarthy, Roger E. Schecter \& David J. Franklyn, McCarthy's Desk Encyclopedia of Intellectual Property, 312 ( $3^{\text {rd }}$ ed., Bureau of National Affairs, Bloomberg, Washington, 2004).

115 Entendida como la facultad positiva de realizar actos sobre el objeto; el facere o facere solus del que habla Hermenegildo Baylos-Corroza, Tratado de derecho industrial, 283 (3 ${ }^{\text {a ed., }}$ Civitas, Navarra, 2009).

116 Carlos Fernández-Novoa, José Manuel Otero-Lastres \& Manuel Botana-Agra, Manual de la propiedad industrial, 161 (Marcial Pons, Madrid, 2009). 
distribución, semejante al reconocido por el derecho de autor ${ }^{117}$; o bien, afirmando que el agotamiento de los derechos de propiedad industrial afecta a las facultades que en un orden lógico siguen a la puesta en el comercio del bien que materializa o que incorpora el DPI, como sostiene José Massaguer-Fuentes "en particular, el ofrecimiento, introducción en comercio, utilización, posesión con los fines anteriores y, en su caso, importación con tales fines de los productos puestos en el comercio"118. El agotamiento en consecuencia no afectará ni a la facultad de prohibir la fabricación del producto objeto de la patente, ni a la de prohibir la utilización o el ofrecimiento de utilización del procedimiento objeto de la patente, ni a la de prohibir la entrega u ofrecimiento de entrega de medios relativos a un elemento esencial de la invención patentada que son necesarios para su puesta en práctica ${ }^{119}$.

En el caso del derecho de autor - aunque el derecho objeto del agotamiento está identificado plenamente, esto es, el derecho de distribución-, tal claridad enunciativa ${ }^{120}$ no corresponde con el ejercicio práctico del agotamiento de derechos de autor; en especial

117 Juan David Castro-García, El agotamiento de los derechos de propiedad intelectual, 13 Revista La Propiedad Inmaterial, 253-282, 257 (2009). Disponible en: http://revistas.uexternado.edu. co/index.php/propin/article/view/463/3631

118 José Massaguer-Fuentes, El contenido y alcance del derecho de patente, Actualidad Jurídica Uría Menéndez, Homenaje al profesor D. Rodrigo Uría González en el centenario de su nacimiento, número extraordinario, 173-187, 181 (2006). Disponible en: http://www.uria.com/documentos/ publicaciones/1632/documento/art12.pdf?id=2158

119 José Massaguer-Fuentes, El contenido y alcance del derecho de patente, Actualidad Jurídica Uría Menéndez, Homenaje al profesor D. Rodrigo Uría González en el centenario de su nacimiento, número extraordinario, 173-187 (2006). Disponible en: http://www.uria.com/documentos/ publicaciones/1632/documento/art12.pdf?id=2158

120 Estados Unidos, Copyright Act, 17 USCS Sects. 101-810, 1976, art. 109. Disponible en: http:// www.copyright.gov/title17/, http://copyright.gov/title17/92appa.pdf, https://www.law.cornell. edu/copyright/copyright.table.html. Unión Europea, Directiva 96/9/CE del Parlamento Europeo y del Consejo, sobre la protección jurídica de las bases de datos, 11 de marzo de 1996. Disponible en: http://www.wipo.int/wipolex/es/details.jsp?id=1409. Unión Europea, Directiva 2001/29/CEE del Parlamento Europeo, relativa a la armonización de algunos aspectos del derecho de autor en la sociedad de la información, 22 de mayo de 2001. Disponible en: http://www. wipo.int/wipolex/es/details.jsp?id=1453. Unión Europea, Directiva 2006/115/CE del Parlamento Europeo y del Consejo sobre derechos de alquiler y préstamo y otros derechos afines a los derechos de autor en el ámbito de la propiedad intelectual (versión codificada), 12 de diciembre de 2006. Disponible en: http://www.wipo.int/wipolex/es/details.jsp?id=5815. Unión Europea, Directiva 2009/24/CE del Parlamento Europeo y del Consejo sobre la protección jurídica de programas de ordenador (versión codificada), 23 de abril de 2009. Disponible en: http://www. wipo.int/wipolex/es/details.jsp?id=8612. Organización Mundial de Propiedad Intelectual, Tratado de la OMPI sobre Derecho de Autor (WIPO Copyright Treaty, WCT), Ginebra, 20 de diciembre de 1996. Disponible en: http://www.wipo.int/treaties/es/text.jsp?file_id=295167, aplicable para Estados Unidos, la Unión Europea y Colombia. 
luego de la sentencia de la Corte de Justicia de la Unión Europea en el caso UsedSoft. En este asunto, la Corte reconoció que luego de la primera "disposición" (venta o licencia) hecha respecto del soporte digital de una obra protegida, cualquier uso o aprovechamiento individual posterior (reventa, relicenciamiento o puesta a disposición) implica necesariamente el uso de nuevas facultades diferentes a la simple distribución (por ejemplo, la reproducción, la comunicación pública o la puesta a disposición) ${ }^{121}$.

De esta forma, quienes afirman que resulta imposible aplicar el agotamiento de los derechos de autor para el caso de las obras digitales, sostienen que en el mercado digital no es posible simplemente distribuir una obra, toda vez que cualquier aprovechamiento digital, incluso el que está destinado a traspasar un soporte digital de una persona a otra, implica el uso de algún otro derecho de autor ${ }^{122}$; por ejemplo, la reproducción ${ }^{123}$. También hay quienes justifican una interpretación ajustada al entorno digital, no mutatis mutandis ${ }^{124}$ desde los supuestos fácticos analógicos, sino ejerciendo el principio de equivalencia funcional ${ }^{125}$ con el propósito de hacer posibles los efectos analógicos en los digitales ${ }^{126}$. O quienes buscan asegurar intereses generales ${ }^{127}$, como la permanencia de todos los límites y

121 En la sentencia UsedSoft se evidencia el conflicto entre las directivas 2001/29/CE y 2009/24/ CE. Tribunal de Justicia de la Unión Europea, TJUE, UsedSoft GmbH v. Oracle International Corp. C-128/11 (3 de julio de 2012). Disponible en: http://curia.europa.eu/juris/document/ document.jsf?docid $=124564 \&$ doclang $=$ en

122 Ricardo Antequera-Parilli, Estudios de derecho industrial y derecho de autor, 595 (1 ${ }^{\mathrm{a}}$ ed., Temis, Bogotá, 2009).

123 YeCID ANDRÉs Ríos-Pinzón, El agotamiento del derecho de autor y los derechos conexos (contenido, alcance y aplicación a las transmisiones en línea). Disponible en el Centro Colombiano del Derecho de Autor, CECOLDA: http://www.cecolda.org.co/index.php/informacion/ columnista-invitado/262-el-agotamiento-del-derecho-de-autor-y-los-derechos-conexoscontenido-alcance-y-aplicacion-a-las-transmisiones-en-linea

124 Lukas Feiler sí habla de un first download como desarrollo del first sale. LuKas FeILER, Birth of the First-Download Doctrine: The Application of the First-Sale Doctrine to Internet Downloads under EU and U.S. Copyright Law (Transatlantic Technology Law Forum Working Papers, February, 2013). Disponible en: http://law.stanford.edu/wp-content/uploads/sites/default/files/ publication/378203/doc/slspublic/feiler_wp17.pdf

125 Principio mediante el cual se extienden los efectos de validez, normalmente otorgados al mundo analógico, hasta los soportes digitales, a fin de otorgar seguridad jurídica al comercio electrónico. María Clara Gutiérrez-Gómez, Consideraciones sobre el tratamiento jurídico del comercio electrónico, 13 (1 ${ }^{\mathrm{a}}$ ed., Legis, Bogotá, 2005).

126 Louise Longdin \& Phen Hoon Lim, Inexhaustible Distribution Rights for Copyright Owners and the Foreclosure of Secondary Markets for Software, 44 IIC, International Review of Intellectual Property and Competition Law, 5, 541-568 (2013).

127 En este sentido, la figura del agotamiento del derecho se ha identificado como una herramienta de política pública. Shubнa Grosh, The Implementation of Exhaustion Policies: Lessons from National Experiences (University of Wisconsin Legal Studies Research Paper Series 1248, 
excepciones en favor de los usuarios de DPI aun en el ámbito digital, por medio de figuras contractuales como las licencias implícitas para ciertos actos de comercialización dentro de un mercado ${ }^{128}$. Es decir, mediante la regulación de ciertos contratos relacionados con la propiedad intelectual, en los que el otorgamiento de una licencia respecto de un tipo de facultad lleva implícita otra $u$ otras licencias sobre facultades diferentes, pero necesarias para llevar a cabo los usos garantizados por el interés general antes mencionado.

Finalmente, respecto a los derechos agotados se debe analizar el alcance de la figura; toda vez que se ha discutido si una vez agotado el DPI, tal agotamiento conlleva una carga u obligación para el titular de permitir al usuario la disposición de su ejemplar, así como el correlativo derecho de este último para hacer valer su condición de propietario del ejemplar.

Tal discusión no trae mayor dificultad cuando el análisis está circunscrito a copias tangibles del objeto de propiedad intelectual; sin embargo, al desplazar el análisis a los soportes digitales, la pregunta que surge ${ }^{129}$ es si el titular de DPI debe garantizar que el usuario, a favor de quien se agotó el derecho, disfrute de aquellas prerrogativas que el agotamiento, en principio, garantiza: la disposición de ese ejemplar.

Al respecto solo se ha encontrado respuesta en la jurisprudencia alemana $^{130}$, que decidió que el titular de derechos de propiedad intelectual no está obligado a garantizar tales prerrogativas a los usuarios; más aún, el titular, que no puede impedir el agotamiento de su derecho, sí puede impedir la libre disposición de los usuarios con medidas tecnológicas. Por ejemplo, imponiendo medidas

2004). Disponible en: http://papers.ssrn.com/sol3/papers.cfm?abstract_id=2390232

128 Orit Fischman-Afori, Implied License - An Emerging New Standard in Copyright Law, 25 Santa Clara Computer and High Technology Law Journal, 275-325 (2008). Disponible en: http:// ssrn.com/abstract $=1266083$

129 Orit Fischman-Afori, Implied License - An Emerging New Standard in Copyright Law, 25 Santa Clara Computer and High Technology Law Journal, 275-325 (2008). Disponible en: http:// ssrn.com/abstract $=1266083$

130 Alemania, Corte Suprema alemana, Decisión BGH, I ZR 178/08 - Half-Life2 (Bundesgerichtshof, BGH, 11 de febrero de 2010). Disponible en: http://dejure.org/dienste/vernetzung/ rechtsprechung?Text $=\mathrm{I} \% 20 \mathrm{ZR} \% 20178 / 08$. En este caso, el titular del software ató su uso a una cuenta on-line, la cual él mismo determinó como intransferible, de forma que el usuario registrado de la cuenta (y el software) era el único habilitado para usar el software. De esta forma, se restringe técnicamente el uso de software de segunda mano. 
tecnológicas de restricción que inhabilitan el acceso al objeto de propiedad intelectual protegido (software). 


\section{CONCLUSIONES}

- La figura del agotamiento de los derechos de propiedad intelectual tiene un claro origen jurisprudencial en Estados Unidos y la Unión Europea. Esta jurisprudencia, además, evidencia las teorías que explicaban la propiedad intelectual para cada momento histórico. De forma que para finales del siglo IX y principios del siglo XX, serán las teorías utilitarias de la propiedad las que justifiquen la propiedad intelectual y la misma figura del agotamiento de los DPI que, a lo largo de toda su evolución, ha sido entendida como una limitación intrínseca a la propiedad intelectual.

A mediados del siglo XX, "el mercado" irrumpió con gran fuerza como un elemento de análisis dentro del agotamiento de los DPI. En este momento, se hizo una clara asociación entre la figura del agotamiento con el control de los mercados secundarios de productos protegidos por la propiedad intelectual; y es con la ayuda del análisis propio de la competencia (al que se suma de forma complementaria el derecho de contratos) que se determinará si las restricciones posventa afectan o no al mercado. La inclusión del factor "mercado" en lo que tradicionalmente era un ámbito exclusivo de la propiedad, ha llevado a discutir sobre la conveniencia de permitir la yuxtaposición de las que antes eran tres categorías diferenciadas: (i) los derechos de propiedad intelectual; (ii) las relaciones contractuales; y (iii) los efectos en la libre competencia. Incluso, hay quienes afirman que las explicaciones basadas en los análisis de los mercados han llevado al decaimiento de figuras como el agotamiento del derecho, es decir, el desuso de un límite a la propiedad intelectual.

- Más allá de las relaciones del agotamiento con otros factores (determinantes o no), las discusiones en torno a la figura no se han caracterizado por tratar de comprobar si la figura del agotamiento pertenece a una u otra categoría específica en los derechos de propiedad intelectual. Es decir, esta figura a lo largo de su desarrollo ha sido tratada como límite a la propiedad intelectual, independientemente de su cercanía, o no, con otros factores como el mercado o los contratos. 
- Hoy, la doctrina se acerca masivamente a discusión sobre el objeto del agotamiento de los DPI y su acoplamiento al mundo digital. Los derechos que resultan susceptibles del agotamiento y los tipos de soportes respecto de los cuales el titular de derechos se desprende de sus facultades, son el centro de la discusión que obtienen distintas respuestas dependiendo de los factores que se incorporen al análisis de la situación.

- De otra parte, la figura del agotamiento resulta ligada al comercio internacional (más allá del comunitario) solamente como una consecuencia, en la medida en que el surgimiento, consolidación y desarrollo de la figura han correspondido a los ordenamientos internos (y su propia concepción de interés general — en relación con la identificación de la figura con los límites a los derechos de propiedad-); además, cuando las normas internacionales se han visto abocadas a regular la figura del agotamiento de los DPI han terminado por dejar en absoluta libertad a cada uno de los países para desarrollar su alcance y regulación.

En este sentido, la figura del agotamiento en Colombia, que como hemos comprobado carece de un origen jurisprudencial (tanto interno como comunitario), no parece tener una justificación sólida que explique la regulación de la materia. 


\section{BIBLIOGRAFÍA}

\section{Libros}

Antequera-Parilli, Ricardo, Estudios de derecho industrial y derecho de autor (1 ${ }^{\text {a }}$ ed., Temis, Bogotá, 2009).

Baylos-Corroza, Hermenegildo, Tratado de derecho industrial (3 $3^{\mathrm{a}}$ ed., Civitas, Navarra, 2009).

Bohannan, Christina \& Hovenkamp, Herbert, Creation without Restraint. Promoting Liberty and Rivalry in Innovation (Oxford University Press, Oxford, 2012).

CARbajo-CASCón, Fernando, La distribución selectiva y el comercio paralelo de productos de lujo (1 ${ }^{\text {a }}$ ed., Grupo Editorial Ibáñez, Bogotá, 2010).

Cass, Ronald A. \& Hylton, Keith N., The Laws of Creation. Property Rights in the World of Ideas (Harvard University Press, Boston, 2013).

Chijane-Dapkevicius, Diego, Derecho de marcas. Función y concepto, nulidades, registro, representación gráfica. Derecho comparado ( $1^{\mathrm{a}} \mathrm{ed}$., Editorial B de F, Montevideo, 2011).

Fernández-Novoa, Carlos; Otero-Lastres, José Manuel \& Botana-Agra, Manuel, Manual de la Propiedad Industrial (Marcial Pons, Madrid, 2009).

Gutiérrez-Gómez, María Clara, Consideraciones sobre el tratamiento jurídico del comercio electrónico (1 $1^{\text {a }}$ ed., Legis, Bogotá, 2005).

Hovenkamp, Herbert J., Innovation and Competition Policy, Ch. 10 (2 $2^{\text {nd }}$ ed.): Post-Sale and Related Distribution Restraints Involving IP Rights, Cases and Materials on Innovation and Competition Policy, Chapter 10 (2013). Disponible en: http:// papers.ssrn.com/sol3/papers.cfm?abstract_id=1949392

KoHler, Josef, Handbuch des Deutschen Patentrechts in Rechtsvergleichender Darstellung ( $1^{\text {st }}$ ed., Druck und Verlag von J. Bensheimer, Mannheim, 1900).

Marzorati, Osvaldo J., Sistemas de distribución comercial. Contratos de agencia, distribución, concesión y franchising. Modalidades de instrumentación. Normativa aplicable. Derechos y obligaciones de las partes. Renuncia. Rescisión. Solidaridad laboral. Defensa de la competencia. Internet. Propiedad industrial. Criterios jurisprudenciales. Tendencias en el derecho extranjero ( $3^{\mathrm{a}}$ ed., Editorial Astrea, Buenos Aires, 2011).

McCarthy, J. Thomas; Schecter, Roger E. \& Franklyn, David J., McCarthy's Desk Encyclopedia of Intellectual Property ( $3^{\text {rd }}$ ed., Bureau of National Affairs, Bloomberg, Washington, 2004).

Merges, Robert P., Justifying Intellectual Property (Harvard University Press, Cambridge, London, 2011).

Patry, William, How to Fix Copyright (Oxford University Press, Oxford, 2011).

Peña-Quiñonez, Ernesto, El derecho de bienes (1 $1^{\text {a }}$ ed., Librería Jurídicas Wilches, Bogotá, 1995). Disponible en: http://www.dasumo.com/libros/el-derecho-debienes- $\%$ E2 $\% 80 \% 93$-ernesto-pe $\% \mathrm{C} 3 \%$ Bla-qui $\% \mathrm{C} 3 \% \mathrm{~B} 1$ onez-pdf.html 
Tritton, Guy, Intellectual Property in Europe (3 ${ }^{\text {rd }}$ ed., Sweet \& Maxwell, London, 2008).

Zapata de Arbeláez, Adriana, comp., Derecho Internacional de los Negocios. Alcances (1 ${ }^{\text {a }}$ ed., Universidad Externado de Colombia, Bogotá, 2009).

\section{Contribución en obras colectivas}

Ruiz-Peris, Juan Ignacio, Agotamiento comunitario de la marca y agotamiento internacional en la doctrina del Tribunal de Justicia, en El papel de la jurisprudencia en el TJCE en la armonización del derecho europeo - situación y perspectiva tras cincuenta años, 353-392 ( $1^{\text {a }}$ ed., SAlvador Vilata-Menadas, coord., Universitat de València, València, 2005).

Velandia, Mauricio, Agotamiento del derecho de marca y su relación con el derecho de la competencia, en Derecho internacional de los negocios. Alcances, 427-442 (1 ed., Adriana Zapata de Ar belÁez, comp., Universidad Externado de Colombia, Bogotá, 2009)

\section{Revistas}

Barragán, Carolina; Ceballos, Miguel; Marín, Diana \& Tamayo, Óscar, Agotamiento del derecho a la luz del derecho comunitario. Unión Europea y Comunidad Andina, 16 Revista La Propiedad Inmaterial, 225-280 (2012). Disponible en: http://revistas. uexternado.edu.co/index.php/propin/article/view/3271/3680

Bohannan, Christina, Copyright Preemption of Contracts, 67 Maryland Law Review, 611-663 (2008). Disponible en: http://papers.ssrn.com/sol3/papers.cfm?abstract_ id $=1138251$

Castro-García, Juan David, El agotamiento de los derechos de propiedad intelectual, 13 Revista La Propiedad Inmaterial, 253-282 (2009). Disponible en: http://revistas. uexternado.edu.co/index.php/propin/article/view/463/3631

Cottier, Thomas, The Prospects for Intellectual Property in GATT, 28 Common Market Law Review, 2, 383-414 (1991).

Even, Yonatan, Appropriability, First Sale \& Exhaustion, 1-77 (September 28, 2008). Disponible en: http://ssrn.com/abstract $=1274822$

Fischman-Afori, Orit, Implied License - An Emerging New Standard in Copyright Law, 25 Santa Clara Computer and High Technology Law Journal, 275-325 (2008). Disponible en: http://ssrn.com/abstract $=1266083$

Fisher, William W., III, The Growth of Intellectual Property: A History of the Ownership of Ideas in the United States/Geistiges Eigentum - ein ausufernder Rechtsbereich: Die Geschichte des Ideenschutzes in den Vereinigten Staaten, Eigentum im Internationalen Vergleich, 265-291 (Vandenhoeck \& Ruprecht, 1999). Disponible en: http://cyber.law.harvard.edu/people/tfisher/iphistory.pdf

Goldberg, Melissa, A Textbook Dilemma: Should the First Sale Doctrine Provide a Valid Defense for Foreign-Made Goods?, 81 Fordham Law Review, 101-136 (2012). Disponible en: http://ssrn.com/abstract $=2042811$ 
Harn-Lee, Yin, UsedSoft GmbH v. Oracle International Corp (Case C-128/11) - Sales of "Used" Software and the Principle of Exhaustion, 43 IIC, International Review of Intellectual Property and Competition Law, 846-853 (2012).

Harris, Keith, For Promotional Use Only: Is the Resale of a Promotional CD Protected by the First Sale Doctrine?, 30 Cardozo Law Review, 4, 1745-1772 (2009). Disponible en: http://www.cardozolawreview.com/Joomla1.5/content/30-4/HARRIS.30-4. pdf

Hilty, Reto M.; KöKlÜ, Kaya \& Hafendräd, FABian, Software Agreements: Stocktaking and Outlook - Lessons from the UsedSoft v. Oracle Case from a Comparative Law Perspective, 44 International Review of Intellectual Property and Competition Law, 3, 263-292 (2013).

Hovenkamp, Herbert J., Post-Sale Restraints and Competitive Harm: The First Sale Doctrine in Perspective, NYU Annual Survey of American Law, 101-158 (2011). Disponible en: http://papers.ssrn.com/sol3/papers.cfm?abstract_id=1540527\#\#

JAszi, Peter, Toward a Theory of Copyright: The Metamorphoses of 'Authorship', 1991 Duke Law Journal, 455-502 (1991). Disponible en: http://scholarship.law.duke. edu/cgi/viewcontent.cgi?article $=3150 \&$ context $=\mathrm{dlj}$

Longdin, Louise \& Lim, Phen Hoon, Inexhaustible Distribution Rights for Copyright Owners and the Foreclosure of Secondary Markets for Software, 44 IIC, International Review of Intellectual Property and Competition Law, 5, 541-568 (2013).

Massaguer-Fuentes, José, El agotamiento del derecho de marca y las importaciones paralelas, 5 Temas de Derecho Industrial y de la Competencia, 285-328 (2001).

Massaguer-Fuentes, José, El contenido y alcance del derecho de patente, Actualidad Jurídica Uría Menéndez, Homenaje al profesor D. Rodrigo Uría González en el centenario de su nacimiento, número extraordinario, 173-187 (2006). Disponible en: http://www.uria.com/documentos/publicaciones/1632/documento/art12. pdf?id $=2158$

Overdijk, Tueerd; Putt, Polo van der; Vries, Eva de \& Schafft, Thomas, Exhaustion and Software Resale Rights. A Comparison between the European Exhaustion Doctrine and the U.S. First Sale Doctrine in the Light of Recent Case Law, 2 CRi, 33-39 (2011). Disponible en: http://www.vondst-law.com/files/Exhaustion\%20 and $\% 20$ Software $\% 20$ Resale $\% 20$ Rights $\% 20$ CRI\%202011-02.pdf

Perzanowski, Aaron K. \& Schultz, Jason, Digital Exhaustion, 58 University of California, Los Angeles, UCLA Law Review, 889-946 (2011). Disponible en: http:// www.uclalawreview.org/pdf/58-4-1.pdf

Pope, Alexander B., Note: A Second Look at First Sale: An International Look at U.S. Copyright Exhaustion, 19 Journal of Intellectual Property Law, 201-230 (2011).

RuB, Guy A., Rebalancing Copyright Exhaustion, 64 Emory Law Journal, 741-817 (2015). Disponible en: http://law.emory.edu/elj/_documents/volumes/64/3/articles/rub. pdf

Rubí-Puig, Antoni, Copyright Exhaustion Rationales and Used Software: A Law and Economics Approach to Oracle v. UsedSoft, 4 Journal of Intellectual Property, Information Technology and e-Commerce Law, JIPITEC, 3, 159-178 (2013). Disponible en: http://papers.ssrn.com/sol3/papers.cfm?abstract_id=2408659 
Serra, Theodore, Rebalancing at Resale: ReDigi, Royalties, and The Digital Secondary Market, 93 Boston University Law Review, 5, 1753-1801 (2013). Disponible en: http://www.bu.edu/bulawreview/files/2013/10/SERRA_Resale.pdf

Ullrich, Hanns, TRIPS: Adequate Protection, Inadequate Trade, Adequate Competition Policy, 4 Pacific Rim Law \& Policy Journal, 1, 153-210 (1995). Disponible en: http://digital.law.washington.edu/dspace-law/bitstream/handle/1773.1/933/4Pa cRimLPolyJ153.pdf?sequence $=1 \&$ isAllowed $=y$

Uribe-Piedrahíta, Carlos Andrés, Cuestiones concurrenciales y marcarias en la distribución de piezas de recambio (la problemática de los aftermarkets), en Marcas y distribución comercial, 213-268 (EduARdo Galán-Corona \& Fernando CARbajo-CAScón, coords., Ediciones Universidad de Salamanca, Salamanca, 2011).

Westramp, Guido, Intellectual Property, Competition Rules and the Emerging Internal Market: Some Thoughts on the European Exhaustion Doctrine, 11 Marquette Intellectual Property Law Review, 2, 291-335 (2007). Disponible en: http:// scholarship.law.marquette.edu/cgi/viewcontent.cgi?article $=1096 \&$ context $=\mathrm{iplr}$

\section{Working paper, documentos, ponencias}

Feiler, Lukas, Birth of the First-Download Doctrine: The Application of the First-Sale Doctrine to Internet Downloads under EU and U.S. Copyright Law (Transatlantic Technology Law Forum Working Papers, February, 2013). Disponible en: http:// law.stanford.edu/wp-content/uploads/sites/default/files/publication/378203/doc/ slspublic/feiler_wp17.pdf

Grosh, Sнubнa, The Implementation of Exhaustion Policies: Lessons from National Experiences (University of Wisconsin Legal Studies Research Paper Series 1248, 2004). Disponible en: http://papers.ssrn.com/sol3/papers.cfm?abstract id $=2390232$

Hovenkamp, Herbert J., IP and Antitrust Policy: A Brief Historical Overview (University of Iowa Legal Studies Research Paper, 05-31, 2005). Disponible en: http://papers. ssrn.com/sol3/papers.cfm?abstract_id=869417

Hovenkamp, Herbert J., Resale Price Maintenance: Consignment Agreements, Copyrighted or Patented Products and the First Sale Doctrine (University of Iowa Legal Studies Research Paper, 10-42, 2010). Disponible en: http://papers.ssrn. com/sol3/papers.cfm?abstract_id=1671943

Katz, Ariel, What Antitrust Law Can (and Cannot) Teach About the First Sale Doctrine (January 23, 2012). Disponible en: http://papers.ssrn.com/sol3/papers. cfm?abstract_id=1845842

Stothers, Christopher, Patent Exhaustion: the UK Perspective, 16 Annual Conference on Intellectual Property Law and Policy, Fordham University School of Law, 27-28 March 2008. Disponible en: http://fordhamipconference.com/wp-content/ uploads/2010/08/Christopher-Sothers-Patent-Exhaustion.pdf

Torremans, Paul L. C., The Future Implications of the UsedSoft Decision (CREATe Working Paper, 2014). Disponible en: http://zenodo.org/record/8382/files/ CREATe-Working-Paper-2014-02.pdf 


\section{Tratados internacionales}

Convenio de Berna para la Protección de las Obras Literarias y Artísticas, del 9 de septiembre de 1886, completado en París el 4 de mayo de 1896, revisado en Berlín el 13 de noviembre de 1908, completado en Berna el 20 de marzo de 1914 y revisado en Roma el 2 de junio de 1928, en Bruselas el 26 de junio de 1948, en Estocolmo el 14 de julio de 1967, en París el 24 de julio de 1971 y enmendado el 28 de septiembre de 1979. Disponible en: http://www.wipo.int/treaties/es/text. jsp?file_id=283700

Convenio de París para la Protección de la Propiedad Industrial, del 20 de marzo de 1883, revisado en Bruselas el 14 de diciembre de 1900, en Washington el 2 de junio de 1911, en La Haya el 6 de noviembre de 1925, en Londres el 2 de junio de 1934, en Lisboa el 31 de octubre de 1958, en Estocolmo el 14 de julio de 1967 y enmendado el 28 de septiembre de 1979. Disponible en: http://www.wipo.int/ treaties/es/text.jsp?file_id=288515

Organización Mundial de Propiedad Intelectual, Tratado de la OMPI sobre Derecho de Autor (WIPO Copyright Treaty, WCT), Ginebra, 20 de diciembre de 1996. Disponible en: http://www.wipo.int/treaties/es/text.jsp?file_id=295167

Organización Mundial del Comercio, OMC, Acuerdo por el que se establece la Organización Mundial del Comercio, Marrakech, 15 de abril de 1994. Disponible en: https://www.wto.org/spanish/docs_s/legal_s/legal_s.htm, https://www.wto. org/spanish/docs_s/legal_s/04-wto.pdf

Organización Mundial del Comercio, OMC, Acuerdo sobre los Aspectos de los Derechos de Propiedad Intelectual relacionados con el Comercio (Acuerdo sobre los ADPIC) o Trade-Related aspects of Intellectual Property Rights, TRIPS, Anexo 1C del Convenio por el que se crea la OMC, firmado en 1994. Disponible en: https://www.wto.org/spanish/docs_s/legal_s/legal_s.htm https://www.wto.org/ spanish/docs_s/legal_s/27-trips.pdf

Organización Mundial del Comercio, OMC, Declaración Ministerial de Doha (2001), Doha, Catar, 14 de noviembre de 2001. Disponible en: https://www.wto.org/ spanish/thewto_s/minist_s/min01_s/mindecl_s.htm

Tratado constitutivo de la Comunidad Económica Europea, Tratado CEE, Roma, 25 de marzo de 1957. Disponible en: http://eur-lex.europa.eu/legal-content/ES/ TXT/?uri=URISERV:xy0023

Unión Europea, Tratado de Funcionamiento de la Unión Europea, TFUE, Roma, 25 de marzo de 1957. Versión consolidada disponible en: http://www.boe.es/buscar/ doc.php?id=DOUE-Z-2010-70006

\section{Normatividad internacional}

Comunidad Andina, Decisión Andina 85, que establece el Reglamento para la Aplicación de las Normas sobre Propiedad Industrial (5 de junio de 1974). Disponible en: http://www.wipo.int/wipolex/es/details.jsp?id=9464

Comunidad Andina, Decisión Andina 311, que establece el Régimen Común sobre Propiedad Industrial (8 de noviembre de 1991). Disponible en: http://www.wipo. 
int/wipolex/es/text.jsp?file_id=224231

Comunidad Andina, Decisión Andina 344, que establece el Régimen Común sobre Propiedad Industrial (21 de octubre de 1993). Disponible en: http://www.wipo. int/wipolex/es/details.jsp?id=9454

Comunidad Andina, Decisión Andina 486, que establece el Régimen Común sobre Propiedad Industrial (14 de septiembre de 2000). Disponible en: http://www. wipo.int/edocs/lexdocs/laws/es/can/can012es.pdf

Estados Unidos, Copyright Act, 17 USCS Sects. 101-810, 1976. Disponible en: http:// www.copyright.gov/title17/, http://copyright.gov/title17/92appa.pdf, https://www. law.cornell.edu/copyright/copyright.table.html

Estados Unidos, Patent Act, 1836, Ch. 357, 5 Stat. 117 (July 4, 1836). Disponible en: http://www.ipmall.info/hosted_resources/lipa/patents/patent_act_of_1836.pdf

Estados Unidos, Sherman Act, 15 USC, Sects. 1-7, 1890. Disponible en: http://www. ourdocuments.gov/doc.php?doc $=51 \&$ page $=$ transcript

Unión Europea, Directiva 96/9/CE del Parlamento Europeo y del Consejo, sobre la protección jurídica de las bases de datos, 11 de marzo de 1996. Disponible en: http://www.wipo.int/wipolex/es/details.jsp?id=1409

Unión Europea, Directiva 2001/29/CEE del Parlamento Europeo, relativa a la armonización de algunos aspectos del derecho de autor en la sociedad de la información, 22 de mayo de 2001. Disponible en: http://www.wipo.int/wipolex/ es/details.jsp?id=1453

Unión Europea, Directiva 2006/115/CE del Parlamento Europeo y del Consejo sobre derechos de alquiler y préstamo y otros derechos afines a los derechos de autor en el ámbito de la propiedad intelectual (versión codificada), 12 de diciembre de 2006. Disponible en: http://www.wipo.int/wipolex/es/details.jsp?id=5815

Unión Europea, Directiva 2009/24/CE del Parlamento Europeo y del Consejo sobre la protección jurídica de programas de ordenador (versión codificada), 23 de abril de 2009. Disponible en: http://www.wipo.int/wipolex/es/details.jsp?id=8612

\section{Casos internacionales}

Alemania, Corte Suprema alemana, Decisión BGH, I ZR 178/08 - Half-Life2 (Bundesgerichtshof, BGH, 11 de febrero de 2010). Disponible en: http://dejure. org/dienste/vernetzung/rechtsprechung?Text=I\%20ZR\%20178/08

Alemania, Guajakol-Karbonat. Disponible en: http://opinioiuris.de/entscheidung/1278

Alemania, Kölnisch Wasser (1902).

Alemania, Mariani (1902).

Estados Unidos, Corte de Apelaciones del 2 Circuito, John Wiley \& Sons, Inc. v. Supap Kirtsaeng (US Court of Appeals for the Second Circuit, $2^{\text {nd }}$ Cir. 2011). Disponible en: http://law.justia.com/cases/federal/appellate-courts/ca2/09-4896/09-4896_ complete_opn-2011-08-15.html

Estados Unidos, Corte de Apelaciones del 6 Circuito, Heaton-Peninsular Button-Fastener 
Co. v. Eureka Specialty Co., 77 F. 288 (US Court of Appeals for the Sixth Circuit, $6^{\text {th }}$ Cir. 1896). Disponible en: http://openjurist.org/77/f1d/288

Estados Unidos, Corte de Apelaciones del 6 Circuito, John D. Park \& Sons Co. v. Hartman, 153 F. 24, 27 (US Court of Appeals for the Sixth Circuit, $6^{\text {th }}$ Cir. 1907).

Estados Unidos, Corte de Apelaciones del 7 Circuito, Victor Talking Machine Co. v. The Fair, 123 F. 428 (US Court of Appeals for the Seventh Circuit, $7^{\text {th }}$ Cir. 1903).

Estados Unidos, Corte de Apelaciones del 8 Circuito, National Phonograph Co. v. Schlegel, 128 F. 733 (US Court of Appeals for the Eighth Circuit, $8^{\text {th }}$ Cir. 1904).

Estados Unidos, Corte de Apelaciones del 9 Circuito, MAI Systems Corporation v. Peak Computer, Inc., 991 F.2d 511 (US Court of Appeals for the Ninth Circuit, $9^{\text {th }}$ Cir. 1993). Disponible en: https://www.law.cornell.edu/copyright/cases/991_F2d_511. htm

Estados Unidos, Corte de Apelaciones del 9 Circuito, MDY Industries v. Blizzard Entertainment, No. 09-15932 (US Court of Appeals for the Ninth Circuit, 17 February, 2011). Disponible en: http://cdn.ca9.uscourts.gov/datastore/ opinions/2011/02/17/09-15932.pdf

Estados Unidos, Corte de Apelaciones del 9 Circuito, Vernor v. Autodesk, No. 09-35969 (US Court of Appeals for the Ninth Circuit, 10 September 2010). Disponible en: http://cdn.ca9.uscourts.gov/datastore/opinions/2010/09/10/09-35969.pdf

Estados Unidos, Corte de Apelaciones del Circuito Federal, DSC Communications Corporation v. Pulse Communications, Inc., 170 F.3d 1354 (United States Court of Appeals, Federal Circuit, Fed. Cir. 1999). Disponible en: http://openjurist. org/170/f3d/1354

Estados Unidos, Corte del Distrito Norte de California, División San José, Adobe Systems, Inc. v. Stargate Software Inc., 216 F.Supp.2d 1051 (United States District Court, N.D. California, San Jose Division, N.D. Cal. 2002). Disponible en: https://h2o.law.harvard.edu/cases/4333

Estados Unidos, Corte del Distrito Sur de New York, Capitol Records, LLC v. ReDigi, Inc., No. 12-95, 2013 WL 1286134 (United States District Court, Southern District of New York, SDNY, March 30, 2013). Disponible en: https://cases.justia.com/ federal/district-courts/new-york/nysdce/1:2012cv00095/390216/109/0.pdf

Estados Unidos, Corte Federal del Distrito Norte de Illinois, Mallinckrodt, Inc. v. Medipart, Inc., No. 89-C-4524 (1990) - WL 19535 (Northern District of Illinois Judges, Feb. 16, 1990).

Estados Unidos, Corte Suprema, Adams v. Burke, 84 U.S. 453 (1873). Disponible en: https://supreme.justia.com/cases/federal/us/84/453/case.html

Estados Unidos, Corte Suprema, Aro Manufacturing Co. v. Convertible Top Replacement Co., 365 U.S. 336, 259 (1961). Disponible en: https://supreme.justia.com/cases/ federal/us/365/336/case.html

Estados Unidos, Corte Suprema, Bobbs-Merrill Co. v. Straus, 201 U.S. 339 (1908). Disponible en: http://caselaw.findlaw.com/us-supreme-court/210/339.html

Estados Unidos, Corte Suprema, E. Bement \& Sons v. National Harrow Co., 186 U.S. 70 (1902). Disponible en: https://www.law.cornell.edu/supremecourt/text/186/70 
Estados Unidos, Corte Suprema, Federal Trade Commission v. Actavis, Case No. 12-416 (June 17, 2013). Disponible en: http://www.supremecourt.gov/ opinions/12pdf/12-416_m5n0.pdf

Estados Unidos, Corte Suprema, Keeler v. Standard Folding Bed Co., 157 U.S. 659 (1895). Disponible en: https://supreme.justia.com/cases/federal/us/157/659/case.html

Estados Unidos, Corte Suprema, Quanta Computer, Inc. v. LG Electronics, Inc., $128 \mathrm{~S}$. Ct 2109 (2008). Disponible en: http://www.supremecourt.gov/opinions/07pdf/06-937. pdf

Estados Unidos, Corte Suprema, U.S. v. Line Material Co., 333 U.S. 287, 300 (1948). Disponible en: https://supreme.justia.com/cases/federal/us/333/287/

Estados Unidos, Corte Suprema, U.S. v. Masonite Corporation, 316 U.S. 265, 277-278 (1942). Disponible en: https://supreme.justia.com/cases/federal/us/316/265/case. html

Estados Unidos, Corte Suprema, U.S. v. Univis Lens Co., 316 U.S. 241 (1942). Disponible en: https://supreme.justia.com/cases/federal/us/316/241/case.html

Inglaterra y Gales, Corte de Apelaciones de Londres, División Civil, Michael Baigent y Richard Leigh contra The Random House Group Limited [2007] EWCA Civ 247, Caso A3 2006/0971, Código Da Vinci, jueces John Mummery, Bernard Rix y Anthony Lloyd. Disponible en: http://www.bailii.org/ew/cases/EWCA/ Civ/2007/247.html

Tribunal de Justicia de la Comunidad Andina, Proceso 2-AI-96 (20 de junio de 1997). Disponible en: http://intranet.comunidadandina.org/Documentos/ DocumentosEntrada/E00000622011.pdf

Tribunal de Justicia de la Comunidad Andina, Proceso 17-IP-98 (21 de abril de 1998). Disponible en: http://intranet.comunidadandina.org/documentos/ DocumentosEntrada/E00012222011.pdf

Tribunal de Justicia de la Comunidad Andina, Proceso 24-IP-2005 (25 de mayo de 2005). Disponible en: http://intranet.comunidadandina.org/documentos/ DocumentosEntrada/E00015142011.pdf

Tribunal de Justicia de las Comunidades Europeas, TJCE, Central Farm contra Sterling Drug (1974).

Tribunal de Justicia de las Comunidades Europeas, TJCE, Central Farm contra Winthrop (1974).

Tribunal de Justicia de las Comunidades Europeas, TJCE, Deutsche Grammophon, GmbH contra Metro-SB-Grossmarket, GmbH \& Co., asunto 78/70 (1971). Disponible en: http://www.ippt.eu/files/1971/IPPT19710608_ECJ_Deutche_ Grammophon_Gesellschaft_v_Metro.pdf

Tribunal de Justicia de las Comunidades Europeas, TJCE, Foreningen af danske Videogramdistributører, FDV v. Laserdisken, C-61/97, Rec. p. I-5171, apartado 13 (22 de septiembre de 1998. Disponible en: http://curia.europa.eu/juris/showPdf. jsf?docid $=44104 \&$ doclang $=E S$

Tribunal de Justicia de las Comunidades Europeas, TJCE, IHT Internationale Heiztechnik GmbH v. Ideal-Standard GmbH, E.C.R. I-2789 (1994). Disponible 
en: http://curia.europa.eu/juris/liste.jsf?num=C-9/93

Tribunal de Justicia de las Comunidades Europeas, TJCE, Keurkoop BV v. Nancy Kean Gift BV, E.C.R. 2853 (1982). Disponible en: http://biicl.org/files/2026_c-144-81.pdf

Tribunal de Justicia de las Comunidades Europeas, TJCE, Merck \& Co. v. Stephar BV, E.C.R. 2063 (1981). http://www.ippt.eu/files/1981/IPPT19810714_ECJ_Merck_v_ Stephar.pdf

Tribunal de Justicia de las Comunidades Europeas, TJCE, Metronome Musik GmbH v. Music Point Hokamp GmbH, C-200/96, Rec. p. I-1953 (28 de abril de 1998). Disponible en: http://dejure.org/dienste/vernetzung/ rechtsprechung?Text=C-200/96

Tribunal de Justicia de las Comunidades Europeas, TJCE, Musik-Vertrieb Membran GmbH v. GEMA, E.C.R. 147 (1981). Disponible en: http://www.ippt.eu/files/1981/ IPPT19810120_ECJ_Membran_and_K-Tel_v_GEMA.pdf

Tribunal de Justicia de las Comunidades Europeas, TJCE, Pharmon BV v. Hoechst AG, E.C.R. 2281 (1985). Disponible en: http://www.ippt.eu/files/1985/IPPT19850709_ ECJ_Pharmon.pdf

Tribunal de Justicia de las Comunidades Europeas, TJCE, Sirena S.r.l. v. Eda S.r.l., caso 40/70 (1971).

Tribunal de Justicia de la Unión Europea, TJUE, Football Association Premier League y otros v. QC Leisure y otros, C-403/08 y C-429/08 (4 de octubre de 2011). Disponible en: http://curia.europa.eu/juris/liste.jsf?num=C-403/08\&language $=$ en

Tribunal de Justicia de la Unión Europea, TJUE, UsedSoft GmbH v. Oracle International Corp. C-128/11 (3 de julio de 2012). Disponible en: http://curia.europa.eu/juris/ document/document.jsf?docid=124564\&doclang $=$ en

\section{Jurisprudencia colombiana}

Tribunal Superior de Bogotá, Sala Civil, Luis Alejandro Velasco contra Gabriel García Márquez, 27 de enero de 1994, magistrado ponente Ricardo Zopó-Méndez.

\section{Publicaciones de internet}

MAdrid, Luis Ángel, Importaciones paralelas. Agotamiento del derecho de propiedad intelectual (Universidad Sergio Arboleda, octubre 2005). Disponible en: http:// www.usergioarboleda.edu.co/tlc/tlc_importaciones\%20paralelas.htm

Organización Mundial de Propiedad Intelectual, OMPI, Glosario de términos. Disponible en: http://www.wipo.int/wipolex/en/glossary/\#O

Organización Mundial del Comercio, OMC, Glosario de términos. Disponible en: http:// www.wto.org/spanish/thewto_s/glossary_s/glossary_s.htm, https://www.wto.org/ english/thewto_e/glossary_e/glossary_e.htm

Ríos-Pinzón, Yecid Andrés, El agotamiento del derecho de autor y los derechos conexos (contenido, alcance y aplicación a las transmisiones en línea). Disponible en el 
Centro Colombiano del Derecho de Autor, CECOLDA: http://www.cecolda. org.co/index.php/informacion/columnista-invitado/262-el-agotamiento-delderecho-de-autor-y-los-derechos-conexos-contenido-alcance-y-aplicacion-alas-transmisiones-en-linea 
